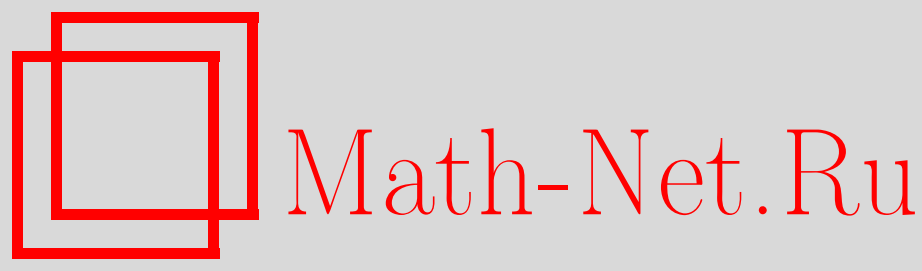

Х. С. Ниров, А. В. Разумов, О $\mathbb{Z}$-градуированных алгебрах Ли петель, группах петель и уравнениях Тоды, TMФ, 2008, том 154, номер 3, 451-476

DOI: https://doi.org/10.4213/tmf6181

Использование Общероссийского математического портала Math-Net.Ru подразумевает, что вы прочитали и согласны с пользовательским соглашением http://www . mathnet.ru/rus/agreement

Параметры загрузки:

IP: 54.166 .219 .16

26 апреля 2023 г., 02:11:25

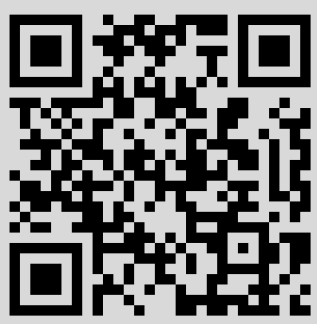




\section{О Z्Z-ГРАДУИРОВАННЫХ АЛГЕБРАХ ЛИ ПЕТЕЛЬ, ГРУППАХ ПЕТЕЛЬ И УРАВНЕНИЯХ ТОДЫ}

Рассмотрены уравнения Тоды, ассоциированные со скрученными группами петель. Такие уравнения определяются $\mathbb{Z}$-градуировками соответствующих скрученных алгебр Ли петель. Обсуждается классификация уравнений Тоды, которые связаны со скрученными алгебрами Ли петель, снабженными интегрируемыми $\mathbb{Z}$-градуировками.

Ключевые слова: уравнения Тоды, группы петель, $\mathbb{Z}$-градуированные алгебры Ли петель.

\section{1. ВВЕДЕНИЕ}

Уравнение Тоды - это матричное дифференциальное уравнение специального вида, эквивалентное некоторому набору нелинейных дифференциальных уравнений второго порядка. Оно ассоциировано с группой Ли и определяется $\mathbb{Z}$-градуировкой соответствующей алгебры Ли [1]-[3]. Представляют определенный интерес обобщения тодовских систем, возникающие при включении высших градуировочных подпространств [4]-[7] и при переходе в пространства более высоких размерностей [8], [9]. Упомянем, кроме того, работы [10], [11], в которых аффинные тодовские системы рассматриваются как двухпетлевые калибровочные модели ВЗНВ с потенциальным членом.

Групповая и алгебраическая классификация уравнений Тоды, ассоциированных с конечномерными комплексными классическими группами Ли, была проведена в работах [12]-[14], где эти уравнения были записаны в удобной блок-матричной форме, порождаемой рассматриваемой $\mathbb{Z}$-градуировкой.

Алгебро-групповые и дифференциально-геометрические свойства тодовских систем и их физический смысл существенно различаются в зависимости от того, с какой группой Ли - конечномерной или бесконечномерной - они ассоциированы. Поучительным примером яляются два простейших частных случая тодовских си-

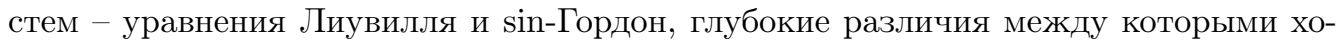
рошо изучены. В общем случае при работе с группами Ли петель имеют дело с

* Институт ядерных исследований РАН, Москва, Россия. E-mail: nirov@ms2.inr.ac.ru

${ }^{\dagger}$ Институт физики высоких энергий, Протвино, Московская обл., Россия 
бесконечномерными многообразиями [15], что может приводить к дополнительным проблемам по сравнению с конечномерным случаем. Так, при использовании произвольной $\mathbb{Z}$-градуировки алгебры Ли петель нужно иметь в виду возможные расходимости бесконечных рядов градуировочных компонент. Тщательный анализ групп петель комплексных простых групп Ли и соответствующих $\mathbb{Z}$-градуированных алгебр Ли петель был проведен в [16]. В частности, в этой работе было введено весьма полезное понятие интегрируемых $\mathbb{Z}$-градуировок. Впоследствии в работе [17] на основе этого рассмотрения были явно описаны уравнения Тоды, ассоциированные с группами петель комплексных классических групп Ли. Здесь мы даем краткий обзор работ [16], [17], опуская доказательства и сосредотачиваясь в основном на логике исследования.

\section{2. УРАВНЕНИЯ ТОДЫ, АССОЦИИРОВАННЫЕ С ГРУППАМИ ЛИ ПЕТЕЛЬ}

2.1. Общее определение уравнения Тоды. В настоящей работе $\mathcal{M}$ означает либо евклидову плоскость $\mathbb{R}^{2}$, либо комплексную прямую $\mathbb{C}$. Мы обозначаем стандартные координаты на $\mathbb{R}^{2}$ через $z^{-}$и $z^{+}$. Те же обозначения используются для стандартной комплексной координаты на $\mathbb{C}$ и комплексно сопряженной координаты: $z=z^{-}$и $\bar{z}=z^{+}$соответственно. Как обычно, $\partial_{-}=\partial / \partial z^{-}$и $\partial_{+}=\partial / \partial z^{+}$.

Напомним, что алгебра Ли $\mathfrak{G}$ называется $\mathbb{Z}$-градуированной, если задано представление $\mathfrak{G}$ в виде прямой суммы подпространств $\mathfrak{G}_{k}$ таких, что

$$
\left[\mathfrak{G}_{k}, \mathfrak{G}_{l}\right] \subset \mathfrak{G}_{k+l}
$$

для любых $k, l \in \mathbb{Z}$. Это означает, что любой элемент $\xi$ алгебры $\mathfrak{G}$ может быть единственным образом представлен как

$$
\xi=\sum_{k \in \mathbb{Z}} \xi_{k}
$$

где $\xi_{k} \in \mathfrak{G}_{k}$ для любого $k \in \mathbb{Z}$. В случае, когда $\mathfrak{G}$ является бесконечномерной алгеброй Ли, мы полагаем, что она наделена структурой топологического векторного пространства и приведенный выше ряд сходится абсолютно.

Пусть дана группа Ли $\mathcal{G}$, и ее алгебра Ли $\mathfrak{G}$ наделена $\mathbb{Z}$-градуировкой. Предположим, что для некоторого положительного целого числа $L$ градуировочные подпространства $\mathfrak{G}_{-k}$ и $\mathfrak{G}_{+k}$ тривиальны при всех $0<k<L$. Согласно определению $\mathbb{Z}$-градуировки ее градуировочное подпространство $\mathfrak{G}_{0}$, соответствующее нулевому градуировочному индексу, является подалгеброй $\mathfrak{G}$, и мы обозначаем через $\mathcal{G}_{0}$ связную подгруппу Ли группы $\mathcal{G}$, соответствующую этой подалгебре.

Уравнение Тодъ, ассоциированное с группой Ли $\mathcal{G},-$ это нелинейное матричное дифференциальное уравнение второго порядка для гладкого отображения $\Xi$ из $\mathcal{M}$ 
в $\mathcal{G}_{0}$ следующего явного вида ${ }^{1)}$ :

$$
\partial_{+}\left(\Xi^{-1} \partial_{-} \Xi\right)=\left[\mathcal{F}_{-}, \Xi^{-1} \mathcal{F}_{+} \Xi\right]
$$

(см., в частности, книги [1], [3]). В этом уравнении $\mathcal{F}_{-}$и $\mathcal{F}_{+}-$некоторые фиксированные отображения из $\mathcal{M}$ в $\mathfrak{G}_{-L}$ и $\mathfrak{G}_{+L}$ соответственно, удовлетворяющие условиям

$$
\partial_{+} \mathcal{F}_{-}=0, \quad \partial_{-} \mathcal{F}_{+}=0 .
$$

Если группа Ли $\mathcal{G}_{0}$ абелева, то говорят, что соответствующее уравнение Тоды абелево; в противном случае мы имеем дело с неабелевым уравнением Тоды. Напомним также, что в рамках дифференциально-геометрического подхода уравнение Тоды может быть получено из условия нулевой кривизны плоской связности на тривиальном главном расслоении $\mathcal{M} \times \mathcal{G} \rightarrow \mathcal{M}$ путем наложения определенных градуировочных и калибровочных условий [3].

Авторы статьи [10] рассматривают уравнения вида (1), предполагая, что не существует $L$ для которого подпространства $\mathfrak{G}_{-k}$ и $\mathfrak{G}_{+k}$ тривиальны при $0<k<L$. На самом деле, такое предположение не приводит к новым уравнениям Тоды. Действительно, рассмотрим подалгебру $\mathfrak{G}^{\prime}$ алгебры $\mathfrak{G}$, определенную как

$$
\mathfrak{G}^{\prime}=\bigoplus_{k \in \mathbb{Z}} \mathfrak{G}_{k L},
$$

и соответствующую подгруппу $\mathcal{G}^{\prime}$ группы $\mathcal{G}$. Разложение $(3)$ определяет $\mathbb{Z}$-градуировку $\mathfrak{G}^{\prime}$ с градуировочными подпространствами $\mathfrak{G}_{k}^{\prime}=\mathfrak{G}_{k L}, k \in \mathbb{Z}$. Очевидно, что уравнение Тоды, ассоциированное с группой Ли $\mathcal{G}$ и с таким выбором положительного целого числа $L$, может быть рассмотрено как уравнение Тоды, ассоциированное с группой Ли $\mathcal{G}^{\prime}$ и выбором $L=1$.

Если задан изоморфизм $F$ из $\mathbb{Z}$-градуированной алгебры Ли $\mathfrak{G}$ в $\mathbb{Z}$-градуированную алгебру Ли $\mathfrak{H}$, связывающий соответствующие градуировочные подпространства равенством $\mathfrak{H}_{k}=F\left(\mathfrak{G}_{k}\right)$, то говорят, что $\mathbb{Z}$-градуировки $\mathfrak{G}$ и $\mathfrak{H}$ сопряжены посредством $F$. Действительно, имея $\mathbb{Z}$-градуированную алгебру Ли $\mathfrak{G}$, можно индуцировать $\mathbb{Z}$-градуировку $F$-изоморфной алгебры Ли $\mathfrak{H}$, используя $\mathfrak{H}_{k}=F\left(\mathfrak{G}_{k}\right)$ в качестве градуировочных подпространств.

Ясно, что сопряженные $\mathbb{Z}$-градуировки дают практически одни и те же уравнения Тоды. Поэтому для классификации уравнений Тоды, ассоциированных с группой Ли $\mathcal{G}$, нужно построить классификацию несопряженных $\mathbb{Z}$-градуировок ее алгебры Ли $\mathfrak{G}$. Для случая, когда $\mathfrak{G}$ является комплексной классической группой Ли, удобная классификация $\mathbb{Z}$-градуировок была описана в работе [14] (см. также работы [12], [13]). В настоящей статье мы приводим обзор и обсуждение соответствующих результатов, полученных в статьях [16], [17] для случая, когда $\mathcal{G}$ является группой петель комплексной классической группы Ли.

\footnotetext{
1) Для простоты мы считаем, что $\mathcal{G}$ является подгруппой группы, образованной обратимыми элементами некоторой ассоциативной алгебры $\mathcal{A}$ с единицей. В этом случае $\mathfrak{G}$ может быть рассмотрена как подалгебра алгебры Ли, ассоциированной с $\mathcal{A}$. На самом деле, наше рассмотрение может быть обобщено на случай произвольной группы $\mathcal{G}$.
} 
2.2. Алгебры Ли петель и группы петель. Пусть $\mathfrak{g}$ - конечномерная вещественная или комплексная алгебра Ли. Алгебра Ли петель алгебры g, обозначаемая через $\mathcal{L}(\mathfrak{g})$, обычно определяется как линейное пространство $C^{\infty}\left(S^{1}, \mathfrak{g}\right)$-гладких отображений единичной окружности $S^{1}$ в $\mathfrak{g}$ с законом умножения алгебры Ли, задаваемым поточечно. В данной статье мы определяем $\mathcal{L}(\mathfrak{g})$ как линейное пространство $C_{2 \pi}^{\infty}(\mathbb{R}, \mathfrak{g})$-гладких $2 \pi$-периодических отображений вещественной прямой $\mathbb{R}$ в $\mathfrak{g}$ также с поточечным определением операции умножения в алгебре Ли. Можно показать, что эти две алгебры Ли изоморфны. Предположим, что $\mathcal{L}(\mathfrak{g})$ снабжена структурой пространства $\Phi$ реше ${ }^{2)}$, так что операция умножения алгебры Ли является непрерывной (см., например, [16], [18], [19]). Мы называем такую алгебру Ли алгеброй Лu-Фреше.

Пусть теперь дана группа Ли $G$ с алгеброй Ли g. Груnпа nетель группы $G$, обозначаемая $\mathcal{L}(G)$, определяется либо как множество $C^{\infty}\left(S^{1}, G\right)$-гладких отображений из $S^{1}$ в $G$, либо как множество $C_{2 \pi}^{\infty}(\mathbb{R}, G)$-гладких $2 \pi$-периодических отображений из $\mathbb{R}$ в $G$ с законом группового умножения, определенным в обоих случаях поточечно. В настоящей работе мы принимаем второе определение. Предположим, что $\mathcal{L}(G)$ снабжена структурой многообразия Фреше, моделируемого на $\mathcal{L}(\mathfrak{g})$ таким образом, что она становится группой Ли (см., например, [16], [18], [19]). При этом алгебра Ли группы Ли $\mathcal{L}(G)$ естественно отождествляется с алгеброй Ли петель $\mathcal{L}(\mathfrak{g})$.

Нетрудно обобщить данные выше определения на случай скрученных алгебр Ли петель и групп петель. Пусть $A$ является автоморфизмом алгебры Ли $\mathfrak{g}$, удовлетворяющим для некоторого положительного целого числа $M$ условию $A^{M}=\mathrm{id}_{\mathfrak{g}}$. Cкрученная алгебра Ли петель $\mathcal{L}_{A, M}(\mathfrak{g})$ - это подалгебра алгебры Ли петель $\mathcal{L}(\mathfrak{g})$, образованная элементами $\xi$, которые удовлетворяют равенству $\xi(\sigma+2 \pi / M)=A(\xi(\sigma))$. Аналогично для заданного автоморфизма $a$ группы Ли $G$, который удовлетворяет соотношению $a^{M}=\operatorname{id}_{G}$, определим скрученную группу петель $\mathcal{L}_{a, M}(G)$ как подгруппу группы петель $\mathcal{L}(G)$, образованную элементами $\rho$ такими, что $\rho(\sigma+2 \pi / M)=$ $a(\rho(\sigma))$. Алгебра Ли скрученной группы петель $\mathcal{L}_{a, M}(G)$ естественно отождествляется со скрученной алгеброй Ли петель $\mathcal{L}_{A, M}(\mathfrak{g})$, где автоморфизм алгебры Ли $\mathfrak{g}$, соответствующий автоморфизму а группы Ли $G$, обозначен через $A$.

Понятно, что алгебра Ли петель $\mathcal{L}(\mathfrak{g})$ может рассматриваться как скрученная алгебра Ли петель $\mathcal{L}_{\mathrm{id}_{\mathfrak{g}}, M}(\mathfrak{g})$, где $M$ является произвольным положительным целым числом. В свою очередь группа петель $\mathcal{L}(G)$ может рассматриваться как скрученная группа петель $\mathcal{L}_{\mathrm{id}_{G}, M}(G)$, где $M$ снова есть произвольное положительное целое число. В настоящей статье под алгебрами Ли петель и группами петель мы подразумеваем скрученные алгебры Ли петель и скрученные группы петель.

\section{3. $\mathbb{Z}$-градуировки алгебр Ли петель и соответствующие уравнения То-} ды. Сначала напомним метод, использованный в работе [14] для описания $\mathbb{Z}$-градуировок алгебр Ли комплексных классических групп Ли. Такими группами мы называем группы Ли $G L_{n}(\mathbb{C}), O_{n}(\mathbb{C})$ и $S p_{n}(\mathbb{C})$, алгебрами Ли которых являются $\mathfrak{g l}_{n}(\mathbb{C}), \mathfrak{s o}_{n}(\mathbb{C})$ и $\mathfrak{s p}_{n}(\mathbb{C})$ соответственно (см. п. 3.1).

2) Пространство Фреше - это полное топологическое векторное пространство, топология которого индуцирована счетным семейством полунорм. 
Начнем с немного более широкого класса алгебр Ли. Пусть $\mathfrak{G}$ - конечномерная комплексная простая алгебра Ли, снабженная $\mathbb{Z}$-градуировкой. Определим линейный оператор $Q$, действующий на элемент $\xi \in \mathfrak{G}$ следующим образом:

$$
Q \xi=\sum_{k \in \mathbb{Z}} k \xi_{k}
$$

где $\xi_{k}, k \in \mathbb{Z}$, являются градуировочными компонентами $\xi$. Ясно, что

$$
\mathfrak{G}_{k}=\{\xi \in \mathfrak{G} \mid Q \xi=k \xi\}
$$

Таким образом, оператор $Q$ полностью определяет соответствующую $\mathbb{Z}$-градуировку. Он называется градуировочным оператором, порождающим рассматриваемую $\mathbb{Z}$-градуировку.

Можно легко показать, что $Q$ является дифференцированием алгебры Ли $\mathfrak{G}$. Хорошо известно, что любое дифференцирование комплексной простой алгебры Ли является внутренним. Следовательно, существует единственный элемент $q \in \mathfrak{G}$ такой, что $Q \xi=[q, \xi]$. Это значит, что проблема классификации $\mathbb{Z}$-градуировок $\mathfrak{G}$ в рассматриваемом случае сводится к проблеме классификации элементов $q \in \mathfrak{G}$, для которых оператор $\operatorname{ad}(q)$ является полупростым и имеет только целочисленные собственные значения. Известно, что любой такой элемент принадлежит некоторой подалгебре Картана алгебры Ли $\mathfrak{G}$. Поскольку все подалгебры Картана сопряжены посредством внутренних автоморфизмов алгебры Ли $\mathfrak{G}$, то для того чтобы классифицировать $\mathbb{Z}$-градуировки алгебры $\mathfrak{G}$ с точностью до сопряжений, можно считать, что элемент $q$ принадлежит некоторой фиксированной подалгебре Картана.

Если $\mathfrak{G}$ является комплексной классической алгеброй Ли, то удобно работать с подалгеброй Картана, образованной диагональными матрицами. При этом проблема классификации $\mathbb{Z}$-градуировок алгебры $\mathfrak{G}$ становится совсем простой, и ее результаты могут быть наглядно представлены с помощью блок-матричного разложения элементов $\mathfrak{G}$, которое оказывается очень удобным для описания тодовских систем, ассоциированных с комплексными классическими группами Ли [14].

Главный урок состоит в том, что полезно описывать $\mathbb{Z}$-градуировки алгебры Ли $\mathfrak{G}$ посредством их градуировочных операторов, являющихся специальным случаем дифференцирований алгебры $\mathfrak{G}$. В случае бесконечномерных алгебр Ли-Фреше нужно иметь в виду, что в определение дифференцирования включено требование непрерывности. Если от этого требования отказаться, то представляется невозможным получить содержательные результаты относительно вида дифференцирований.

Пусть теперь $\mathcal{G}$ является бесконечномерной группой Ли-Фреше, а $\mathfrak{G}$ - ее алгеброй Ли-Фреше. В случае общей $\mathbb{Z}$-градуировки алгебры $\mathfrak{G}$ невозможно использовать соотношение (4) для определения линейного оператора в $\mathfrak{G}$, так как ряд в правой части этого соотношения может расходиться для некоторых $\xi$. Мы говорим, что рассматриваемая $\mathbb{Z}$-градуировка порождается градуировочным оператором, если этот ряд сходится абсолютно для любого $\xi \in \mathfrak{G}$. 
Для $\mathfrak{G}=\mathcal{L}_{A, M}(\mathfrak{g})$ основным примером является стандартная $\mathbb{Z}$-градуировка, порождаемая градуировочным оператором $Q=-i d / d s$. Ее градуировочными подпространствами являются

$$
\mathcal{L}_{A, M}(\mathfrak{g})_{k}=\left\{\xi \in \mathcal{L}_{A, M}(\mathfrak{g}) \mid \xi=e^{i k s} x, x \in \mathfrak{g}, A(x)=e^{2 \pi i k / M} x\right\} .
$$

Предположим, что $\mathfrak{G}$ снабжена $\mathbb{Z}$-градуировкой, которая порождается градуировочным оператором $Q$. Можно показать, что тогда

$$
Q[\xi, \eta]=[Q \xi, \eta]+[\xi, Q \eta]
$$

для любых $\xi, \eta \in \mathfrak{G}$. Это означает, что если градуировочный оператор непрерывен, то он является дифференцированием $\mathfrak{G}$.

Ограничим далее рассмотрение случаем, когда $\mathfrak{G}$ является алгеброй Ли петель $\mathcal{L}_{A, M}(\mathfrak{g})$ конечномерной комплексной простой алгебры Ли $\mathfrak{g}$. Предположим, что $\mathcal{L}_{A, M}(\mathfrak{g})$ снабжена $\mathbb{Z}$-градуировкой, порождаемой непрерывным градуировочным оператором $Q$, который является дифференцированием $\mathcal{L}_{A, M}(\mathfrak{g})$. Дифференцирования $\mathcal{L}_{A, M}(\mathfrak{g})$ могут быть описаны явно [16], и это дает ${ }^{3)}$

$$
Q \xi=-i X(\xi)+i[\eta, \xi]
$$

где $X$ - гладкое $(2 \pi / M)$-периодическое комплексное векторное поле на $\mathbb{R}$, а $\eta$ элемент $\mathcal{L}_{A, M}(\mathfrak{g})$. Для того чтобы продвинуться дальше, желательно показать, что $X$ является вещественным векторным полем. Это можно сделать, если ограничиться так называемыми интегрируемыми $\mathbb{Z}$-градуировками [16].

Мы называем $\mathbb{Z}$-градуировку алгебры Ли-Фреше $\mathfrak{G}$ интегрируемой, если отображение $\Phi: \mathbb{R} \times \mathfrak{G} \rightarrow \mathfrak{G}$, определенное соотношением

$$
\Phi(\tau, \xi)=\sum_{k \in \mathbb{Z}} e^{-i k \tau} \xi_{k}
$$

является гладким. Здесь, как обычно, через $\xi_{k}$ мы обозначаем градуировочные компоненты элемента $\xi$. Любая такая градуировка порождается непрерывным градуировочным оператором $Q$, действующим на элемент $\xi \in \mathfrak{G}$ следующим образом:

$$
Q \xi=\left.i \frac{d}{d t}\right|_{0} \Phi_{\xi} .
$$

В этом соотношении $t$ есть стандартная координата на $\mathbb{R}$, а гладкое отображение $\Phi_{\xi}: \mathbb{R} \rightarrow \mathfrak{G}$ определяется равенством $\Phi_{\xi}(\tau)=\Phi(\tau, \xi)$. Кроме того, для любого фиксированного $\tau \in \mathbb{R}$ отображение $\Phi_{\tau}: \xi \in \mathfrak{G} \mapsto \Phi(\tau, \xi)$ является автоморфизмом $\mathfrak{G}$, и все такие автоморфизмы образуют однопараметрическую подгруппу группы автоморфизмов алгебры $\mathfrak{G}$.

Вернемся опять к алгебре Ли петель $\mathcal{L}_{A, M}(\mathfrak{g})$, где $\mathfrak{g}$ является конечномерной комплексной простой алгеброй Ли, и предположим, что она снабжена интегрируемой

\footnotetext{
3)Знаки в правой части уравнения (5) выбраны для удобства дальнейшего рассмотрения. Эта же причина объясняет и появление там мнимой единицы.
} 
$\mathbb{Z}$-градуировкой. Используя явный вид автоморфизмов алгебры Ли $\mathcal{L}_{A, M}(\mathfrak{g})[16]$ и равенство (6), можно убедиться, что в этом случае градуировочный оператор $Q$ определен соотношением (5), где векторное поле $X$ вещественно. Можно показать при этом, что векторное поле $X$ либо является нулевым векторным полем, либо вообще не имеет нулей [16]. Если $X$ есть нулевое векторное поле, то некоторые из градуировочных подпространств оказываются бесконечномерными. Ограничимся $\mathbb{Z}$-градуировками с конечномерными градуировочными подпространствами. Тогда можно считать, что функция $X(s)$, где $s$ - стандартная координата на $\mathbb{R}$, является положительной. Действительно, если $X(s)$ отрицательна, то можно сопрячь рассматриваемую $\mathbb{Z}$-градуировку изоморфизмом, переводящим элемент $\xi \in \mathcal{L}_{A, M}(\mathfrak{g})$ в элемент $\xi^{\prime} \in \mathcal{L}_{A^{-1}, M}(\mathfrak{g})$ такой, что $\xi^{\prime}(\sigma)=\xi(-\sigma)$. Ясно, что это преобразование обращает знак векторного поля $X$.

Теперь, используя сопряжения изоморфизмами, попытаемся привести градуировочный оператор $Q$, задаваемый соотношением (5), к наиболее простому виду. Для этой цели рассмотрим отображение $F$ из $\mathcal{L}_{A, M}(\mathfrak{g})$ в $C^{\infty}(\mathbb{R}, \mathfrak{g})$, определенное равенством

$$
F \xi=\rho\left(f^{-1 *} \xi\right) \rho^{-1},
$$

где $f$ - диффеоморфизм $\mathbb{R}$, а $\rho$ - элемент $C^{\infty}(\mathbb{R}, G)$. Отображение $F$ инъективно и может быть рассмотрено как изоморфизм из $\mathcal{L}_{A, M}(\mathfrak{g})$ в $F\left(\mathcal{L}_{A, M}(\mathfrak{g})\right)$. Можно показать, что

$$
F Q F^{-1} \xi=-i X^{\prime}(\xi)+i\left[\rho \eta^{\prime} \rho^{-1}+X^{\prime}(\rho) \rho^{-1}, \xi\right],
$$

где $X^{\prime}=f_{*} X$ и $\eta^{\prime}=f^{-1 *} \eta$.

Выберем диффеоморфизм $f$ так, чтобы выполнялось равенство $f_{*} X=d / d s$. Для этого достаточно определить его соотношением

$$
f(\sigma)=\int_{(0, \sigma)} \frac{d s}{X(s)} .
$$

Здесь важно, что векторное поле $X$ не имеет нулей. Поскольку векторное поле $X$ является $2 \pi / M$-периодичным, то

$$
f\left(\sigma+\frac{2 \pi}{M}\right)=f(\sigma)+\frac{2 \pi}{M^{\prime}},
$$

где $M^{\prime}$ - положительное вещественное число такое, что

$$
\frac{2 \pi}{M^{\prime}}=\int_{(0,2 \pi / M)} \frac{d s}{X(s)} .
$$

Это равенство означает, в частности, что

$$
\eta^{\prime}\left(\sigma+\frac{2 \pi}{M^{\prime}}\right)=A\left(\eta^{\prime}(\sigma)\right) .
$$

Предположим теперь, что отображение $\rho$ является решением уравнения

$$
\rho^{-1} \frac{d \rho}{d s}=-\eta^{\prime} .
$$


Хорошо известно, что это уравнение всегда имеет решения, все его решения гладкие, и если $\rho$ и $\rho^{\prime}$ суть два решения, то $\rho^{\prime}=g \rho$ для некоторого $g \in G$. Из соотношения (7) следует, что если $\rho$ является решением уравнения (8), то отображение $\rho^{\prime}$, определенное равенством $\rho^{\prime}(\sigma)=a^{-1}\left(\rho\left(\sigma+2 \pi / M^{\prime}\right)\right)$, тоже есть решение уравнения (8). Следовательно, для некоторого $g \in G$ имеем

$$
\rho\left(\sigma+\frac{2 \pi}{M^{\prime}}\right)=a(g \rho(\sigma)) .
$$

Нетрудно убедиться в том, что для описанного выше выбора $f$ и $\rho$ отображение $F$ отображает $\mathcal{L}_{A, M}(\mathfrak{g})$ изоморфно на алгебру Ли-Фреше $\mathfrak{G}$, образованную гладкими отображениями $\xi$ из $\mathbb{R}$ в $\mathfrak{g}$, удовлетворяющими условию

$$
\xi\left(\sigma+\frac{2 \pi}{M^{\prime}}\right)=A^{\prime}(\xi(\sigma)),
$$

где автоморфизм $A^{\prime}$ определяется как $A^{\prime}=A \circ \operatorname{Ad}(g)$. Градуировочный оператор $F Q F^{-1}$, порождающий сопряженную градуировку алгебры $\mathfrak{G}$, есть просто $-i d / d s$. Нетрудно показать [16], что $M^{\prime}$ является целым числом и что $A^{\prime M^{\prime}}=\mathrm{id}_{\mathfrak{g}}$. Это значит, что $\mathfrak{G}=\mathcal{L}_{A^{\prime}, M^{\prime}}(\mathfrak{g})$.

Итак, мы видим, что любая интегрируемая $\mathbb{Z}$-градуировка алгебры Ли петель $\mathcal{L}_{A, M}(\mathfrak{g})$ с конечномерными градуировочными подпространствами сопряжена посредством изоморфизма стандартной $\mathbb{Z}$-градуировке другой скрученной алгебры Ли петель $\mathcal{L}_{A^{\prime}, M^{\prime}}(\mathfrak{g})$; при этом автоморфизмы $A$ и $A^{\prime}$ различаются на внутренний автоморфизм $\mathfrak{g}$. Напомним, что мы рассматриваем случай, когда $\mathfrak{g}$ является конечномерной комплексной простой алгеброй Ли.

Пусть даны конечномерная комплексная простая группа Ли $G$, автоморфизм $a$ группы $G$, удовлетворяющий соотношению $a^{M}=\mathrm{id}_{G}$, и соответствующий автоморфизм $A$ алгебры Ли $\mathfrak{g}$ группы $G$. Для классификации уравнений Тоды, ассоциированных с $\mathcal{L}_{a, M}(G)$, необходимо построить классификацию (с точностью до сопряжений изоморфизмами) $\mathbb{Z}$-градуировок $\mathcal{L}_{A, M}(\mathfrak{g})$. Как мы показали выше, если ограничиться интегрируемыми $\mathbb{Z}$-градуировками с конечномерными градуировочными подпространствами, то эта задача становится эквивалентной классификации самих групп петель $\mathcal{L}_{a, M}(G)$ или, что то же самое, классификации автоморфизмов конечного порядка группы $G$. Нетрудно убедиться [17], что последнюю классификацию достаточно провести также с точностью до сопряжений изоморфизмами. В действительности мы будем классифицировать автоморфизмы конечного порядка алгебры Ли $\mathfrak{g}$, которые могут быть подняты до автоморфизмов группы Ли $G$.

Важно понимать, что каждый автоморфизм $A$ алгебры Ли $\mathfrak{g}$, удовлетворяющий соотношению $A^{M}=\mathrm{id}_{\mathfrak{g}}$, индуцирует $\mathbb{Z}_{M}$-градуировку $\mathfrak{g}$ с градуировочными подпространствами $^{4)}$

$$
\mathfrak{g}_{[k]_{M}}=\left\{x \in \mathfrak{g} \mid A(x)=e^{2 \pi i k / M} x\right\}, \quad k=0, \ldots, M-1 .
$$

И наоборот, любая $\mathbb{Z}_{M}$-градуировка $\mathfrak{g}$ определяет очевидным образом автоморфизм $A$ алгебры $\mathfrak{g}$, удовлетворяющий соотношению $A^{M}=\mathrm{id}_{\mathfrak{g}} \cdot \mathbb{Z}_{M}$-градуировка $\mathfrak{g}$

\footnotetext{
${ }^{4)}$ Мы обозначаем через $[k]_{M}$ элемент кольца $\mathbb{Z}_{M}$, отвечающий целому числу $k$.
} 
называется градуировкой внутреннего или внешнего типа, если ассоциированный с ней автоморфизм $A$ алгебры $\mathfrak{g}$ является автоморфизмом внутреннего или внешнего типа соответственно.

Градуировочные подпространства стандартной $\mathbb{Z}$-градуировки алгебры Ли петель $\mathcal{L}_{A, M}(\mathfrak{g})$ могут быть описаны в терминах соответствующей $\mathbb{Z}_{M}$-градуировки $\mathfrak{g}$ как

$$
\mathcal{L}_{A, M}(\mathfrak{g})_{k}=\left\{\xi \in \mathcal{L}_{A, M}(\mathfrak{g}) \mid \xi=e^{i k s} x, x \in \mathfrak{g}_{[k]_{M}}\right\} .
$$

Для стандартной градуировки положительное целое число $L$, входящее в определение уравнения Тоды, удовлетворяет неравенству $L \leqslant M$, и равенство $L=M$ имеет место тогда и только тогда, когда $A=\mathrm{id}_{\mathfrak{g}}$, при этом положительное целое число $M$ является произвольным. Нетривиальными градуировочными подпространствами являются $\mathcal{L}_{\mathrm{id}_{\mathfrak{g}}, M}(\mathfrak{g})_{k M}$ при $k \in \mathbb{Z}$, и мы имеем

$$
\mathcal{L}_{\mathrm{id}_{\mathfrak{g}}, M}(\mathfrak{g})_{k M}=\left\{\xi \in \mathcal{L}_{\mathrm{id}_{\mathfrak{g}}, M}(\mathfrak{g}) \mid \xi=e^{i k M s} x, x \in \mathfrak{g}\right\} .
$$

Ясно также, что для стандартной $\mathbb{Z}$-градуировки подалгебра $\mathcal{L}_{A, M}(\mathfrak{g})_{0}$ изоморфна $\mathfrak{g}_{[0]_{M}}$, а группа Ли $\mathcal{L}_{a, M}(G)_{0}$ изоморфна связной подгруппе Ли $G_{0}$ группы $G$, соответствующей алгебре Ли $\mathfrak{g}_{[0]_{M}}$. Следовательно, отображение $\Xi$ в действительности является отображением из $\mathcal{M}$ в $G_{0}$; обозначим его $\gamma$, чтобы это соответствовало обозначениям, введенным в более ранних публикациях (см., например, книгу [3]). Отображения $\mathcal{F}_{-}$и $\mathcal{F}_{+}$задаются соотношениями

$$
\mathcal{F}_{-}(p)=e^{-i L s} c_{-}(p), \quad \mathcal{F}_{+}(p)=e^{i L s} c_{+}(p), \quad p \in \mathcal{M},
$$

где $c_{-}$и $c_{+}$являются отображениями из $\mathcal{M}$ в $\mathfrak{g}_{-[L]_{M}}$ и $\mathfrak{g}_{+[L]_{M}}$ соответственно. Таким образом, уравнение Тоды (1) может быть записано в виде

$$
\partial_{+}\left(\gamma^{-1} \partial_{-} \gamma\right)=\left[c_{-}, \gamma^{-1} c_{+} \gamma\right]
$$

где $\gamma$ является гладким отображением из $\mathcal{M}$ в $G_{0}$, а отображения $c_{-}$и $c_{+}$суть фиксированные гладкие отображения из $\mathcal{M}$ в $\mathfrak{g}_{-[L]_{M}}$ и $\mathfrak{g}_{+[L]_{M}}$ соответственно. Условия (2) означают, что

$$
\partial_{+} c_{-}=0, \quad \partial_{-} c_{+}=0 .
$$

Подводя промежуточный итог, можно сказать, что уравнение Тоды, ассоциированное с группой петель простой комплексной группы Ли, алгебра Ли которой наделена интегрируемой $\mathbb{Z}$-градуировкой с конечномерными градуировочными подпространствами, эквивалентно уравнению вида (9).

Здесь имеет смысл отдельно рассмотреть простейший случай, когда $A$ есть $\mathrm{id}_{\mathfrak{g}}$ и $M$ является произвольным положительным целым числом. В этом случае $L=M$. Отображение $\gamma$ является отображением из $\mathcal{M}$ во всю группу $G$, а $c_{+}$и $c_{-}$суть отображения из $\mathcal{M}$ в $\mathfrak{g}$. Положив для этого частного случая $\gamma=\Gamma, c_{+}=C_{+}$и $c_{-}=C_{-}$, запишем уравнение Тоды (9) в виде

$$
\partial_{+}\left(\Gamma^{-1} \partial_{-} \Gamma\right)=\left[C_{-}, \Gamma^{-1} C_{+} \Gamma\right]
$$


а условие (10) - в виде

$$
\partial_{+} C_{-}=0, \quad \partial_{-} C_{+}=0 .
$$

Заметим, что этот пример демонстрирует принципиальное различие между уравнениями Тоды, ассоциированными с конечномерными группами Ли и группами петель.

Можно рассматривать уравнения типа (9) с более общей точки зрения. Пусть $G$ есть произвольная конечномерная группа Ли, и $а$ является произвольным автоморфизмом $G$ конечного порядка. Соответствующий автоморфизм $A$ алгебры Ли $\mathfrak{g}$ группы Ли $G$ порождает $\mathbb{Z}_{M}$-градуировку $\mathfrak{g}$. Предположим, что для некоторого положительного целого числа $L \leqslant M$ градуировочные подпространства $\mathfrak{g}_{+}[k]_{M}$ и $\mathfrak{g}_{-[k]_{M}}$ тривиальны при $0<k<L$. Выберем некоторые фиксированные отображения $c_{+}$ и $c_{-}$из $\mathcal{M}$ в $\mathfrak{g}_{+[L]_{M}}$ и $\mathfrak{g}_{-[L]_{M}}$ соответственно, удовлетворяющие соотношениям (10). Тогда уравнение $(9)$ эквивалентно уравнению Тоды, ассоциированному с группой петель $\mathcal{L}_{a, M}(G)$, алгебра Ли $\mathcal{L}_{A, M}(\mathfrak{g})$ которой наделена стандартной $\mathbb{Z}$-градуировкой.

Отметим, что авторы статьи [11] тоже предлагают использовать уравнение (9) в качестве удобной формы уравнения Тоды, ассоциированного с группой петель. Они не предполагают, что градуировочные подпространства $\mathfrak{g}_{+}[k]_{M}$ и $\mathfrak{g}_{-[k]_{M}}$ тривиальны для $0<k<L$. На самом деле, их допущение не приводит к новым уравнениям Тоды.

Действительно, допустим, что мы не предполагаем тривиальность градуировочных подпространств $\mathfrak{g}_{+[k]_{M}}$ и $\mathfrak{g}_{-[k]_{M}}$ при $0<k<L$. Как и в п. 2.1, рассмотрим подалгебру

$$
\mathfrak{G}^{\prime}=\bigoplus_{k \in \mathbb{Z}} \mathcal{L}_{A, M}(\mathfrak{g})_{k L}
$$

алгебры Ли петель $\mathcal{L}_{A, M}(\mathfrak{g})$ как $\mathbb{Z}$-градуированную алгебру Ли с градуировочными подпространствами $\mathfrak{G}_{k}^{\prime}=\mathcal{L}_{A, M}(\mathfrak{g})_{k L}$. Уравнение Тоды, ассоциированное с группой Ли $\mathcal{L}_{a, M}(G)$ и сделанным выше выбором положительного целого числа $L$, может быть рассмотрено как уравнение Тоды, ассоциированное с группой Ли $\mathcal{G}^{\prime}$, соответствующей алгебре Ли $\mathfrak{G}^{\prime}$, и с выбором $L=1$. Покажем теперь, что алгебра Ли $\mathfrak{G}^{\prime}$ изоморфна некоторой алгебре Ли петель.

Пусть $M^{\prime}$ является наименьшим положительным целым числом, для которого $M^{\prime}[L]_{M}=[0]_{M}$. Другими словами, $M^{\prime}$ является порядком элемента $[L]_{M}$, рассматриваемого как элемент аддитивной группы кольца $\mathbb{Z}_{M}$. Рассмотрим следующую подалгебру $\mathfrak{g}^{\prime}$ алгебры Ли $\mathfrak{g}$ :

$$
\mathfrak{g}^{\prime}=\bigoplus_{k=0}^{M^{\prime}-1} \mathfrak{g}_{k[L]_{M}}
$$

Алгебра Ли $\mathfrak{g}^{\prime}$ может рассматриваться как $\mathbb{Z}_{M^{\prime}}$-градуированная алгебра Ли с градуировочными подпространствами $\mathfrak{g}_{[k]_{M^{\prime}}^{\prime}}^{\prime}=\mathfrak{g}_{k[L]_{M}}$. Обозначим соответствующий автоморфизм $\mathfrak{g}^{\prime}$ через $A^{\prime}$.

Каждый элемент $\xi \in \mathfrak{G}^{\prime}$ может быть представлен в виде абсолютно сходящегося ряда

$$
\xi=\sum_{k \in \mathbb{Z}} e^{i k L s} x_{k}
$$


где $x_{k} \in \mathfrak{g}_{k[L]_{M}}$ для всех $k \in \mathbb{Z}$. Ясно, что ряд

$$
\xi^{\prime}=\sum_{k \in \mathbb{Z}} e^{i k s} x_{k}
$$

сходится абсолютно, и $\xi^{\prime}$ может рассматриваться как элемент алгебры $\mathcal{L}_{A^{\prime}, M^{\prime}}(\mathfrak{g})$. Легко проверить, что отображение, переводящее $\xi$ в $\xi^{\prime}$, является изоморфизмом из $\mathfrak{G}^{\prime}$ в $\mathcal{L}_{A^{\prime}, M^{\prime}}(\mathfrak{g})$.

Итак, если мы не считаем, что градуировочные подпространства $\mathfrak{g}_{+}[k]_{M}$ и $\mathfrak{g}_{-[k]_{M}}$ тривиальны для $0<k<L$, то возникающее уравнение Тоды, ассоциированное с группой петель $\mathcal{L}_{A, M}(G)$, можно трактовать как уравнение Тоды, ассоциированное с некоторой другой группой петель $\mathcal{L}_{A^{\prime}, M^{\prime}}\left(G^{\prime}\right)$, для которого $L=1$. При этом $G^{\prime}$ есть группа Ли, соответствующая подалгебре $\mathfrak{g}^{\prime}$ алгебры Ли $\mathfrak{g}$.

В дальнейшем будет конкретизирован явный вид уравнений Тоды, вытекающих из (9), для случая комплексных классических групп Ли $G$. Ранее было отмечено, что это описание должно использовать классификацию с точностью до сопряжений автоморфизмов конечного порядка рассматриваемых алгебр Ли. Не прибегая к технике корневого разложения, как, например, в [20]-[22], здесь мы проводим такую классификацию в терминах удобных блок-матричных представлений.

\section{3. $\mathbb{Z}_{M}$-ГРАДУИРОВКИ КОМПЛЕКСНЫХ КЛАССИЧЕСКИХ АЛГЕБР ЛИ}

3.1. Комплексные классические группы и алгебры Ли. В данном пункте мы определяем комплексные классические группы Ли и обсуждаем их основные свойства. Дополнительную информацию об этих группах можно найти, например, в работах [17], [20], [22].

Сначала поясним используемые обозначения. Через $I_{n}$ мы обозначаем единичную диагональную матрицу размера $n \times n$, а через $J_{n}$ - единичную симметричную косодиагональную $(n \times n)$-матрицу. Для четного $n$ определим также кососимметричную косодиагональную матрицу размера $n \times n$ :

$$
K_{n}=\left(\begin{array}{cc}
0 & J_{n / 2} \\
-J_{n / 2} & 0
\end{array}\right)
$$

Когда это не приводит к противоречиям, мы пишем вместо $I_{n}, J_{n}$ и $K_{n}$ просто $I, J$ и $K$ соответственно.

Кроме того, примем следующее соглашение. Если $m$ и $B$ являются $(n \times n)$-матрицами, то ${ }^{B} m=B^{-1}{ }^{t} m B$, где ${ }^{\mathrm{t}} m$ - матрица, транспонированная к $m$. Заметим, что ${ }^{J} m$ получается транспонированием $m$ по отношению к ее косой диагонали.

Комплексная общая линейная группа $G L_{n}(\mathbb{C})$ образована всеми невырожденными комплексными $(n \times n)$-матрицами с матричным произведением в качестве группового закона. Алгебра Ли $\mathfrak{g l}_{n}(\mathbb{C})$ группы Ли $G L_{n}(\mathbb{C})$ образована всеми комплексными $(n \times n)$-матрицами с матричным коммутатором в качестве закона умножения алгебры Ли. Группа Ли $G L_{n}(\mathbb{C})$ и алгебра Ли $\mathfrak{g l}_{n}(\mathbb{C})$ не являются простыми. Подгруппа 
$S L_{n}(\mathbb{C})$ группы $G L_{n}(\mathbb{C})$, образованная матрицами с определителем, равным единице, называется комплексной специальной линейной группой. Группа Ли $S L_{n}(\mathbb{C})$ связна и проста. Ее алгебра Ли $\mathfrak{s l}_{n}(\mathbb{C})$ также проста.

Пусть $B$ является комплексной невырожденной $(n \times n)$-матрицей. Элементы $g$ группы $G L_{n}(\mathbb{C})$, выделяемые условием ${ }^{B} g=g^{-1}$, образуют подгруппу Ли группы $G L_{n}(\mathbb{C})$, которая обозначается через $G L_{n}^{B}(\mathbb{C})$. Алгебра Ли $\mathfrak{g l}_{n}^{B}(\mathbb{C})$ группы $G L_{n}^{B}(\mathbb{C})-$ это подалгебра алгебры Ли $\mathfrak{g l}_{n}(\mathbb{C})$, образованная комплексными $(n \times n)$-матрицами $x$, удовлетворяющими условию ${ }^{B} x=-x$.

Для любой симметричной невырожденной $(n \times n)$-матрицы $B$ группа Ли $G L_{n}^{B}(\mathbb{C})$ изоморфна группе Ли $G L_{n}^{J}(\mathbb{C})$. Группа $G L_{n}^{J}(\mathbb{C})$ называется комплексной ортогональной группой и обозначается $O_{n}(\mathbb{C})$. Для элемента $g \in O_{n}(\mathbb{C})$ из равенства $J_{g}=g^{-1}$ получаем, что либо $\operatorname{det} g=1$, либо $\operatorname{det} g=-1$. Элементы $O_{n}(\mathbb{C})$ с определителем, равным единице, образуют связную подгруппу Ли группы $O_{n}(\mathbb{C})$, называемую комплексной специальной ортогональной группой и обозначаемую $S O_{n}(\mathbb{C})$. Эта подгруппа является связной компонентой единицы $O_{n}(\mathbb{C})$. Алгебра Ли группы $S O_{n}(\mathbb{C})$ обозначается $\mathfrak{s o}_{n}(\mathbb{C})$. Ясно, что алгебра Ли группы $O_{n}(\mathbb{C})$ совпадает с алгеброй Ли группы $S O_{n}(\mathbb{C})$. Группа Ли $S O_{n}(\mathbb{C})$ и алгебра Ли $\mathfrak{s o}_{n}(\mathbb{C})$ являются простыми. Специальная ортогональная группа Ли и ее алгебра Ли обладают как внутренними, так и внешними автоморфизмами, причем последние существуют только для четных $n$.

Для четного $n$ возьмем кососимметричную невырожденную $(n \times n)$-матрицу $B$. Тогда группа Ли $G L_{n}^{B}(\mathbb{C})$ изоморфна группе Ли $G L_{n}^{K}(\mathbb{C})$, которая называется комплексной симплектической группой и обозначается $S p_{n}(\mathbb{C})$. Группа Ли $S p_{n}(\mathbb{C})$ связна и проста. Соответствующая алгебра Ли $\mathfrak{s p}_{n}(\mathbb{C})$ тоже проста. Напомним, что симплектическая группа и ее алгебра Ли имеют только внутренние автоморфизмы.

\section{2. $\mathbb{Z}_{M}$-градуировки внутреннего типа комплексных общих линейных} алгебр Ли. В этом пункте мы рассматриваем $\mathbb{Z}_{M}$-градуировки внутреннего типа комплексных общих линейных алгебр Ли. Мы называем такие градуировки градуировками $\mathfrak{g l}_{n}(\mathbb{C})$ типа I. Имеется два других типа $\mathbb{Z}_{M}$-градуировок, порождаемых внешними автоморфизмами комплексных общих линейных алгебр Ли. Они будут рассмотрены в п. 3.4.

Градуировки типа I. Пусть задан внутренний автоморфизм $a$ группы Ли $G L_{n}(\mathbb{C})$, удовлетворяющий соотношению $a^{M}=\mathrm{id}_{G L_{n}(\mathbb{C})}$. Обозначим соответствующий внутренний автоморфизм алгебры Ли $\mathfrak{g l}_{n}(\mathbb{C})$ через $A$. Этот автоморфизм удовлетворяет соотношению $A^{M}=\mathrm{id}_{\mathfrak{g l}}(\mathbb{C})$. Другими словами, $A$ является автоморфизмом $\mathfrak{g l}_{n}(\mathbb{C})$ конечного порядка. Так как мы интересуемся автоморфизмами $\mathfrak{g l}_{n}(\mathbb{C})$ с точностью до сопряжений, то можно предположить, что рассматриваемый автоморфизм $A$ задан соотношением

$$
A(x)=h x h^{-1}
$$

где $h$ - элемент подгруппы $D_{n}(\mathbb{C})$ группы $G L_{n}(\mathbb{C})$, образованной всеми комплексными невырожденными диагональными матрицами (см., например, [17]). Ясно, что при умножении $h$ на произвольное ненулевое комплексное число получается элемент 
подгруппы $D_{n}(\mathbb{C})$, который порождает тот же автоморфизм $\mathfrak{g l}_{n}(\mathbb{C})$, что и исходный элемент.

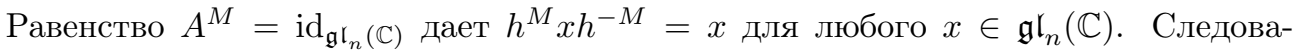
тельно, $h^{M}=\nu I$, где $\nu$ - ненулевое комплексное число. Умножая $h$ на подходящее комплексное число, можно добиться того, что он будет удовлетворять соотношению $h^{M}=I$. Это значит, что диагональные элементы матрицы $h$ будут иметь вид $e^{2 \pi i m / M}$, где $m$ - целое число. Мы будем считать, что $0<m \leqslant M$. Используя внутренние автоморфизмы алгебры $\mathfrak{g l}_{n}(\mathbb{C})$, синхронно переставляющие строки и столбцы матрицы $h$, соберем вместе совпадающие диагональные матричные элементы и придем к следующему блок-диагональному виду элемента $h$ :

$$
h=\left(\begin{array}{llll}
\mu_{1} I_{n_{1}} & & & \\
& \mu_{2} I_{n_{2}} & & \\
& & \ddots & \\
& & & \mu_{p} I_{n_{p}}
\end{array}\right) .
$$

Здесь $\mu_{\alpha}=e^{2 \pi i m_{\alpha} / M}$, положительные целые числа $m_{\alpha}$ образуют убывающую последовательность, $M \geqslant m_{1}>m_{2}>\cdots>m_{p}>0$, и положительные целые числа $n_{\alpha}$ удовлетворяют равенству $\sum_{\alpha=1}^{p} n_{\alpha}=n$. Предполагается, что целое число $p$ больше 1. Случай $p=1$ соответствует $A=\mathrm{id}_{\mathfrak{g l}}(\mathbb{C})$, что приводит к уравнению $(11)$, где $\Gamma$ - отображение из $\mathcal{M}$ в $G L_{n}(\mathbb{C})$, а $C_{-}$и $C_{+}-$отображения из $\mathcal{M}$ в $\mathfrak{g l}_{n}(\mathbb{C})$, удовлетворяющие условиям (12).

Рассмотрим теперь соответствующую $\mathbb{Z}$-градуировку. Представим общий элемент $x$ алгебры $\mathfrak{g l}_{n}(\mathbb{C})$ в блок-матричном виде, который подсказывается структурой $h$ :

$$
x=\left(\begin{array}{cccc}
x_{11} & x_{12} & \ldots & x_{1 p} \\
x_{21} & x_{22} & \ldots & x_{2 p} \\
\vdots & \vdots & \ddots & \vdots \\
x_{p 1} & x_{p 2} & \ldots & x_{p p}
\end{array}\right)
$$

где $x_{\alpha \beta}$ есть $\left(n_{\alpha} \times n_{\beta}\right)$-матрица, $\alpha, \beta=1, \ldots, p$. Легко найти, что

$$
\left(h x h^{-1}\right)_{\alpha \beta}=e^{2 \pi i\left(m_{\alpha}-m_{\beta}\right) / M} x_{\alpha \beta} .
$$

Поэтому если для фиксированных $\alpha$ и $\beta$ только блок $x_{\alpha \beta}$ элемента $x$ отличен от нуля, то $x$ принадлежит градуировочному подпространству $\left[m_{\alpha}-m_{\beta}\right]_{M}$. Удобно ввести целые числа $k_{\alpha}, \alpha=1, \ldots, p-1$, определенные как $k_{\alpha}=m_{\alpha}-m_{\alpha+1}$. По определению для каждого $\alpha$ целое число $k_{\alpha}$ положительно и $\sum_{\alpha=1}^{p-1} k_{\alpha}=m_{1}-m_{p}<M$. Ясно, что для $\alpha<\beta$ имеем $\left[m_{\alpha}-m_{\beta}\right]_{M}=\left[\sum_{\gamma=\alpha}^{\beta-1} k_{\gamma}\right]_{M}$, а для $\alpha>\beta$ имеем $\left[m_{\alpha}-m_{\beta}\right]_{M}=-\left[\sum_{\gamma=\beta}^{\alpha-1} k_{\gamma}\right]_{M}=\left[M-\sum_{\gamma=\beta}^{\alpha-1} k_{\gamma}\right]_{M}$. Эти соотношения позволяют описать градуировочную структуру $\mathbb{Z}_{M}$-градуировки, порождаемой автоморфизмом $A$, с помощью рис. 1. Здесь элементы кольца $\mathbb{Z}_{M}$ - это градуировочные индексы соответствующих блоков в блок-матричном представлении $(15)$ общего элемента $\mathfrak{g l}_{n}(\mathbb{C})$. Заметим, в частности, что подалгебра $\mathfrak{g}_{[0]_{M}}$ образована всеми блок-диагональными 


$\left(\begin{array}{c|c|c|c|c}{[0]_{M}} & {\left[k_{1}\right]_{M}} & {\left[k_{1}+k_{2}\right]_{M}} & \ldots & {\left[\sum_{\alpha=1}^{p-1} k_{\alpha}\right]_{M}} \\ \hline-\left[k_{1}\right]_{M} & {[0]_{M}} & {\left[k_{2}\right]_{M}} & \ldots & {\left[\sum_{\alpha=2}^{p-1} k_{\alpha}\right]_{M}} \\ \hline-\left[k_{1}+k_{2}\right]_{M} & -\left[k_{2}\right]_{M} & {[0]_{M}} & \ldots & {\left[\sum_{\alpha=3}^{p-1} k_{\alpha}\right]_{M}} \\ \hline \vdots & \vdots & \vdots & \ddots & \vdots \\ \hline-\left[\sum_{\alpha=1}^{p-1} k_{\alpha}\right]_{M} & -\left[\sum_{\alpha=2}^{p-1} k_{\alpha}\right]_{M} & -\left[\sum_{\alpha=3}^{p-1} k_{\alpha}\right]_{M} & \cdots & {[0]_{M}}\end{array}\right)$

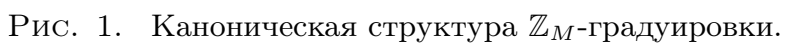

матрицами и изоморфна алгебре Ли $\mathfrak{g l}_{n_{1}}(\mathbb{C}) \times \cdots \times \mathfrak{g l}_{n_{p}}(\mathbb{C})$. Группа $G_{0}$ также образована блок-диагональными матрицами и, следовательно, изоморфна группе Ли $G L_{n_{1}}(\mathbb{C}) \times \cdots \times G L_{n_{p}}(\mathbb{C})$. Такая удобная структура алгебры $\mathfrak{g}_{[0]_{M}}$ и группы $G_{0}$ получена благодаря выбранному упорядочению диагональных матричных элементов $h$. Можно сказать, что числа $\mu_{\alpha}$, взятые как точки на единичной окружности в комплексной плоскости, упорядочены по часовой стрелке.

Ясно, что с точностью до сопряжений $\mathbb{Z}_{M}$-градуировка $\mathfrak{g l}_{n}(\mathbb{C})$ внутреннего типа может быть задана выбором $p \leqslant n$ положительных целых чисел $n_{\alpha}$, удовлетворяющих равенству $\sum_{\alpha=1}^{p} n_{\alpha}=n$, и $p-1$ положительных целых чисел $k_{\alpha}$, удовлетворяющих неравенству $\sum_{\alpha=1}^{p-1} k_{\alpha}<M$. Соответствующий автоморфизм $\mathfrak{g l}_{n}(\mathbb{C})$ определяется соотношением (13), где $h$ задается равенством (14). При этом $\mu_{\alpha}=e^{2 \pi i m_{\alpha} / M}$ и

$$
m_{\alpha}=\sum_{\beta=\alpha}^{p-1} k_{\beta}+m_{p}, \quad \alpha=1, \ldots, p-1,
$$

где $m_{p}$ - произвольное положительное целое число, для которого справедливо неравенство $\sum_{\alpha=1}^{p-1} k_{\alpha}<M$. Различные варианты выбора $m_{p}$ дают один и тот же автоморфизм алгебры $\mathfrak{g l}_{n}(\mathbb{C})$.

\section{3. $\mathbb{Z}_{M}$-градуировки комплексных ортогональных и симплектических} алгебр Ли. С точностью до сопряжений все $\mathbb{Z}_{M}$-градуировки внутреннего и внешнего типа комплексных ортогональных и симплектических алгебр Ли порождаются автоморфизмами, которые задаются соотношением $(13)^{5)}$, где $h$ принадлежит либо группе Ли $O_{n}(\mathbb{C}) \cap D_{n}(\mathbb{C})$, либо группе Ли $S p_{n}(\mathbb{C}) \cap D_{n}(\mathbb{C})$. На самом деле, $O_{n}(\mathbb{C}) \cap D_{n}(\mathbb{C})=S p_{n}(\mathbb{C}) \cap D_{n}(\mathbb{C})$, и удобно предположить, что $h \in O_{n}(\mathbb{C}) \cap D_{n}(\mathbb{C})$. Для любого элемента $x \in \mathfrak{g l}_{n}^{B}(\mathbb{C})$ имеем $h^{M} x h^{-M}=x$, следовательно, $h^{M}=\nu I$ для некоторого комплексного числа $\nu$. Из этого равенства следует, что $\left({ }^{B} h\right)^{M}=\nu I$, и

\footnotetext{
5) Строго говоря, для алгебры Ли $\mathfrak{s o}_{8}(\mathbb{C})$ существуют внешние автоморфизмы, которые не описываются соотношением (13). Однако такие автоморфизмы не могут быть подняты до автоморфизмов группы Ли $S_{8}(\mathbb{C})$ и являются для наших целей несущественными.
} 
поэтому $\left({ }^{B} h\right)^{M} h^{M}=\nu^{2} I$. С другой стороны, используя равенство ${ }^{B} h h=I$, можно получить, что $\left({ }^{B} h\right)^{M} h^{M}=\left({ }^{B} h h\right)^{M}=I$. Отсюда видно, что $\nu^{2}=1$. Другими словами, $\nu= \pm 1$. В обоих случаях диагональные матричные элементы $h$ равны по модулю единице.

Для того чтобы прийти к канонической структуре $\mathbb{Z}_{M}$-градуировки, необходимо привести $h$ к виду (14), где числа $\mu_{\alpha}$ как точки на единичной окружности в комплексной плоскости упорядочены по часовой стрелке. Оказывается, что в некоторых случаях этого невозможно добиться автоморфизмами рассматриваемой алгебры Ли. В этих случаях некоторые из диагональных матричных элементов равны 1 , а некоторые из них равны -1 , и оказывается невозможным собрать такие элементы вместе, оставляя $h$ в $\mathfrak{s o}_{n}(\mathbb{C})$ или $\mathfrak{s} p_{n}(\mathbb{C})$. Может показаться, что похожее препятствие для достижения требуемого упорядочения матричных элементов $h$ возникает и тогда, когда некоторые диагональные матричные элементы равны 1 , даже если там нет матричных элементов, равных -1. Однако в таком случае можно умножить $h$ на -1 , тем самым решая проблему. В результате мы получаем два типа $\mathbb{Z}_{M}$-градуировок комплексных ортогональных и симплектических алгебр Ли.

Градуировки типа I. Начнем со случая, когда оказывается возможным провести желаемое упорядочение чисел $\mu_{\alpha}$, оставаясь внутри $\mathfrak{s o}_{n}(\mathbb{C})$ или $\mathfrak{s p}_{n}(\mathbb{C})$. Как и для $\mathfrak{g l}_{n}(\mathbb{C}), \mathbb{Z}_{M}$-градуировка $\mathfrak{s o}_{n}(\mathbb{C})$ или $\mathfrak{s p}_{n}(\mathbb{C})$ может быть задана посредством выбора $p \leqslant n$ положительных целых чисел $n_{\alpha}$, удовлетворяющих равенству $\sum_{\alpha=1}^{p} n_{\alpha}=n$, и $p-1$ положительных целых чисел $k_{\alpha}$, удовлетворяющих неравенству $\sum_{\alpha=1}^{p-1} k_{\alpha}<M$. При этом очевидно, что эти числа уже не являются столь же произвольными, как в общем линейном случае. Они удовлетворяют дополнительным ограничениям, вытекающим из соответствующих соотношений, задающих группу и алгебру Ли. Конкретно говоря, числа $n_{\alpha}$ удовлетворяют равенствам

$$
n_{p-\alpha+1}=n_{\alpha}, \quad \alpha=1, \ldots, p,
$$

а для чисел $k_{\alpha}$ имеем

$$
k_{p-\alpha}=k_{\alpha}, \quad \alpha=1, \ldots, p-1 .
$$

Пусть $M-\sum_{\alpha=1}^{p-1} k_{\alpha}$ является четным положительным целым числом. Тогда автоморфизм алгебры $\mathfrak{s o}_{n}(\mathbb{C})$ или $\mathfrak{s p}_{n}(\mathbb{C})$, порождающий рассматриваемую $\mathbb{Z}_{M}$-градуировку, определяется соотношением (13), где $h$ задается равенством (14) с $\mu_{\alpha}=$ $e^{2 \pi i m_{\alpha} / M}$. Целое число $m_{p}$ фиксируется равенством

$$
m_{p}=\frac{1}{2}\left(M-\sum_{\alpha=1}^{p-1} k_{\alpha}\right)
$$

а целые числа $m_{\alpha}, \alpha=1, \ldots, p-1$, задаются соотношением (16).

Пусть теперь $M-\sum_{\alpha=1}^{p-1} k_{\alpha}+1$ является четным положительным целым числом. Тогда автоморфизм $\mathfrak{s o}_{n}(\mathbb{C})$ или $\mathfrak{s p}_{n}(\mathbb{C})$, порождающий рассматриваемую $\mathbb{Z}_{M}$-градуировку, определяется соотношением (13), где $h$ задается равенством (14) с $\mu_{\alpha}=$ 
$e^{2 \pi i\left(m_{\alpha}+1 / 2\right) / M}$. Целое число $m_{p}$ фиксируется теперь равенством

$$
m_{p}=\frac{1}{2}\left(M-\sum_{\alpha=1}^{p-1} k_{\alpha}+1\right)
$$

а целые числа $m_{\alpha}, \alpha=1, \ldots, p-1$, снова задаются соотношением (16).

В обоих случаях структура рассматриваемой $\mathbb{Z}_{M}$-градуировки описывается рис. 1 . Однако теперь мы сталкиваемся с необходимостью наложить соответствующие ограничения на блоки представления (15). Это означает, в частности, что алгебра Ли $\mathfrak{g}_{[0]_{M}}$ образована блок-диагональными матрицами. Эта алгебра Ли изоморфна $\mathfrak{g l}_{n_{1}}(\mathbb{C}) \times \cdots \times \mathfrak{g l}_{n_{s}}(\mathbb{C})$ при $p=2 s\left(\right.$ четном $p$ ) и изоморфна $\mathfrak{g l}_{n_{1}}(\mathbb{C}) \times \cdots \times \mathfrak{g l}_{n_{s-1}}(\mathbb{C}) \times$ $\mathfrak{g l}_{n_{s}}^{B_{s}}(\mathbb{C})$ при $p=2 s-1$ (нечетном $p$ ). При этом либо $B_{s}=J_{n_{s}}$, либо $B_{s}=K_{n_{s}}$ в зависимости от того, какая именно алгебра Ли рассматривается. Ясно, что в симплектическом случае для нечетного $p$ целое число $n_{s}$ должно быть четным. Группа Ли $G_{0}$ изоморфна либо $G L_{n_{1}}(\mathbb{C}) \times \cdots \times G L_{n_{s}}(\mathbb{C})$ при $p=2 s$, либо $G L_{n_{1}}(\mathbb{C}) \times \cdots \times G L_{n_{s-1}}(\mathbb{C}) \times$ $G L_{n_{s}}^{B_{s}}(\mathbb{C})$ при $p=2 s-1$.

Градуировки типа II. Этот тип соответствует случаю, когда некоторые из диагональных матричных элементов элемента $h$, порождающего рассматриваемую $\mathbb{Z}_{M}$-градуировку, равны 1 , а некоторые из них равны -1 . Заметим, что такое возможно, только если числа $M$ и $p$ четны.

Переставляя подходящим образом строки и столбцы матриц, представляющих элементы рассматриваемой алгебры Ли, переместим все диагональные матричные элементы $h$, равные 1 , в начало диагонали. Это преобразование может быть осуществлено таким образом, что рассматриваемая алгебра Ли изоморфно отобразится на алгебру Ли $\mathfrak{g l}_{n}^{B}(\mathbb{C})$ с

$$
B=\left(\begin{array}{cc}
J_{n_{1}} & 0 \\
0 & J_{n-n_{1}}
\end{array}\right)
$$

в ортогональном случае и с

$$
B=\left(\begin{array}{cc}
K_{n_{1}} & 0 \\
0 & K_{n-n_{1}}
\end{array}\right)
$$

в симплектическом случае. При этом $n_{1}$ есть число диагональных матричных элементов $h$, равных 1. Затем автоморфизмами алгебры $\mathfrak{g l}_{n}^{B}(\mathbb{C})$ упорядочим остающиеся диагональные матричные элементы $h$ на единичной окружности в комплексной плоскости по часовой стрелке. В результате этих преобразований мы приходим к

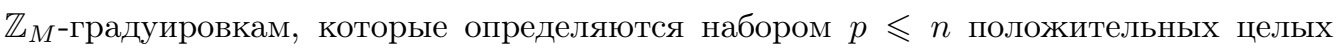
чисел $n_{\alpha}$ таких, что $\sum_{\alpha=1}^{p} n_{\alpha}=n$ и, кроме того,

$$
n_{p-\alpha+2}=n_{\alpha}, \quad \alpha=2, \ldots, p,
$$

и набором $p-1$ положительных целых чисел $k_{\alpha}$ таких, что

$$
\sum_{\alpha=1}^{p-1} k_{\alpha}+k_{1}=M, \quad k_{p-\alpha+1}=k_{\alpha}, \quad \alpha=2, \ldots, p-1 .
$$


Автоморфизм, порождающий рассматриваемую $\mathbb{Z}_{M}$-градуировку, определяется соотношением (13), где $h$ задается равенством (14) с $\mu_{\alpha}=e^{2 \pi i m_{\alpha} / M}$. При этом $m_{p}=k_{1}$, а целые числа $m_{\alpha}, \alpha=1, \ldots, p-1$, задаются соотношением (16).

Структура рассматриваемой $\mathbb{Z}_{M}$-градуировки изображена на рис. 1 . При $p=$ $2 s-2$ алгебра Ли $\mathfrak{g}_{[0]_{M}}$ изоморфна $\mathfrak{g l}_{n_{1}}^{B_{1}} \times \mathfrak{g l}_{n_{2}}(\mathbb{C}) \times \cdots \times \mathfrak{g l}_{n_{s-1}}(\mathbb{C}) \times \mathfrak{g l}_{n_{s}}^{B_{s}}(\mathbb{C})$. Здесь либо $B_{1}=J_{n_{1}}, B_{s}=J_{n_{s}}$, либо $B_{1}=K_{n_{1}}, B_{s}=K_{n_{s}}$ в зависимости от того, какой тип алгебр Ли рассматривается. Ясно, что в симплектическом случае целые числа $n_{1}$ и $n_{s}$ должны быть четными. Группа Ли $G_{0}$ изоморфна $G L_{n_{1}}^{B_{1}}(\mathbb{C}) \times G L_{n_{2}}(\mathbb{C}) \times \cdots$ $\cdots \times G L_{n_{s-1}}(\mathbb{C}) \times G L_{n_{s}}^{B_{s}}(\mathbb{C})$.

\section{4. $\mathbb{Z}_{M}$-градуировки внешнего типа комплексных общих линейных ал-} гебр Ли. Рассмотрение таких градуировок основано на том, что произвольный внешний автоморфизм $A$ алгебры Ли $\mathfrak{g l}_{n}(\mathbb{C})$ сопряжен с автоморфизмом, который определен соотношением

$$
A(x)=-h^{J} x h^{-1},
$$

где $h$ является элементом $G L_{n}(\mathbb{C}) \cap D_{n}(\mathbb{C})$ таким, что ${ }^{J} h h=I$. При этом без потери общности можно предположить, что $\operatorname{det} h=1$. Как и в ортогональном и симплектическом случаях, $h^{M}=\nu I$, где $\nu= \pm 1$. Кроме того, можно убедиться в том, что

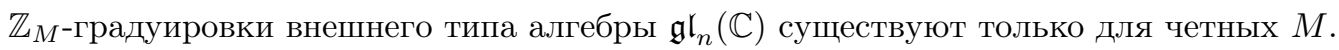
Обозначим $M / 2$ через $N$.

В простейшем случае $h=I$, и существуют только два нетривиальных градуировочных подпространства $\mathfrak{g}_{[0]_{2 N}}$ и $\mathfrak{g}_{[N]_{2 N}}$, элементы которых выделяются условиями ${ }^{J} x=-x$ и ${ }^{J} x=x$ соответственно. В этом случае группа Ли $G_{0}$ совпадает с $S O_{n}(\mathbb{C})$, и мы приходим к уравнению Тоды (11), где $\Gamma$ есть отображение из $\mathcal{M}$ в $S O_{n}(\mathbb{C})$, а $C_{+}$ и $C_{-}$являются отображениями из $\mathcal{M}$ в пространство комплексных $(n \times n)$-матриц $x$, удовлетворяющих равенству ${ }^{J} x=x$.

Для анализа общего случая используют следующие простые соображения. Пусть $B$ есть произвольная невырожденная матрица. Для любого $h \in G L_{n}(\mathbb{C})$ отображение $A: \mathfrak{g l}_{n}(\mathbb{C}) \rightarrow \mathfrak{g l}_{n}(\mathbb{C})$, определенное равенством

$$
A(x)=-h^{B} x h^{-1}
$$

является автоморфизмом алгебры $\mathfrak{g l}_{n}(\mathbb{C})$. Посредством внутреннего автоморфизма $\mathfrak{g l}_{n}(\mathbb{C})$, порожденного элементом $g \in G L_{n}(\mathbb{C})$, автоморфизм $A$ сопряжен с автоморфизмом того же вида, но с заменой $h \rightarrow g h g^{-1}, B \rightarrow{ }^{\mathrm{t}}\left(g^{-1}\right) B g^{-1}$. Заметим также, что при любом $g \in G L_{n}(\mathbb{C})$ замена $h \rightarrow h g, B \rightarrow B g$ не меняет автоморфизм $A$.

Так как $h^{2 N}=\nu I$, где $\nu= \pm 1$, диагональные матричные элементы $h$ по модулю равны единице. Если $\nu=1$, удобно представить каждый из них либо как $e^{2 \pi i m /(2 N)}$, либо как $-e^{2 \pi i m /(2 N)}$, где $m$ - целое число, удовлетворяющее условию $0<m \leqslant N$. При $\nu=-1$ мы используем либо представление $e^{2 \pi i(m+1 / 2) /(2 N)}$, либо представление $-e^{2 \pi i(m+1 / 2) /(2 N)}$, где снова $m$ есть целое число, удовлетворяющее условию $0<m \leqslant N$. Умножая матрицы $h$ и $B$ справа на подходящую матрицу, мы исключаем второй вариант представления. Затем, используя внутренние автоморфизмы алгебры $\mathfrak{g l}_{n}(\mathbb{C})$, мы упорядочиваем диагональные элементы матрицы $h$ удобным 
для наших целей образом. Имеется два различных случая, соответствующих двум дополнительным типам $\mathbb{Z}_{M}$-градуировок $\mathfrak{g l}_{n}(\mathbb{C})$.

Градуировки типа II. Предположим, что ни один из диагональных матричных элементов $h$ не равен 1. Тогда удается привести $h$ к виду $(14)$, где $\mu_{\alpha}=e^{2 \pi i m_{\alpha} /(2 N)}$ при $\nu=1$ или $\mu_{\alpha}=e^{2 \pi i\left(m_{\alpha}+1 / 2\right) /(2 N)}$ при $\nu=-1$. В обоих случаях целые числа $m_{\alpha}$ удовлетворяют неравенствам $0<m_{1}<\cdots<m_{p} \leqslant N$ и соотношениям

$$
m_{p-\alpha+1}=N-m_{\alpha}, \quad \alpha=1, \ldots, p .
$$

Автоморфизм $A$, порождающий рассматриваемую $\mathbb{Z}_{M}$-градуировку, дается теперь уравнением (17), где $B=K_{n}$. Вводя целые числа $k_{\alpha}=m_{\alpha}-m_{\alpha+1}$, легко заметить, что рассматриваемая $\mathbb{Z}_{2 N}$-градуировка $\mathfrak{g l}_{n}(\mathbb{C})$ определяется теми же данными, что и $\mathbb{Z}_{N}$-градуировка типа I соответствующей ортогональной или симплектической алгебры Ли.

Градуировки типа III. Пусть некоторые диагональные матричные элементы $h$ равны 1. Тогда $\nu=1$, и $h$ приводится к виду (14), где $\mu_{\alpha}=e^{2 \pi i m_{\alpha} /(2 N)}$ с $m_{\alpha}$, удовлетворяющими неравенствам $0<m_{1}<\cdots<m_{p} \leqslant N$ и соотношениям

$$
m_{p-\alpha+2}=N-m_{\alpha}, \quad \alpha=2, \ldots, p .
$$

Автоморфизм $A$, порождающий рассматриваемую $\mathbb{Z}_{M}$-градуировку, задается теперь соотношением (17), где $\mathrm{e}^{6}$

$$
B=\left(\begin{array}{cc}
J_{n_{1}} & 0 \\
0 & K_{n-n_{1}}
\end{array}\right) .
$$

При этом $n_{1}$ есть количество диагональных матричных элементов $h$, равных 1 . Вводя целые числа $k_{\alpha}=m_{\alpha}-m_{\alpha+1}$, можно заметить, что рассматриваемая $\mathbb{Z}_{2 N}$-градуировка $\mathfrak{g l}_{n}(\mathbb{C})$ определяется теми же данными, что и $\mathbb{Z}_{N}$-градуировка типа II соответствующей ортогональной или симплектической алгебры Ли.

Наконец, можно показать [17], что $\mathbb{Z}_{2 N}$-градуировки алгебры $\mathfrak{g l}_{n}(\mathbb{C})$ типов II и III могут быть описаны схемой, изображенной на рис. 2. В этой схеме пары элементов кольца $\mathbb{Z}_{2 N}$ суть допустимые градуировочные индексы соответствующих блоков в блок-матричном представлении (15) общего элемента $\mathfrak{g l}_{n}(\mathbb{C})$. Две возможности различаются дополнительными ограничениями, налагаемыми на блоки. А именно, если градуировочный индекс $k$ лежит в пределах $0 \leqslant k<N$, то $x_{\alpha \beta}=-\left({ }^{B} x\right)_{\alpha \beta}$ для $\alpha \leqslant \beta$ и $x_{\alpha \beta}=\left({ }^{B} x\right)_{\alpha \beta}$ для $\alpha>\beta$, тогда как если $k$ находится в пределах $N \leqslant k<2 N$, то $x_{\alpha \beta}=\left({ }^{B} x\right)_{\alpha \beta}$ при $\alpha \leqslant \beta$ и $x_{\alpha \beta}=-\left({ }^{B} x\right)_{\alpha \beta}$ при $\alpha>\beta$.

\section{4. ЯВНЫЙ ВИД УРАВНЕНИЙ ТОДЫ, АССОЦИИРОВАННЫХ С ГРУППАМИ ПЕТЕЛЬ КОМПЛЕКСНЫХ КЛАССИЧЕСКИХ ГРУПП ЛИ}

В данном разделе мы описываем явный вид уравнений Тоды, ассоциированных с группами петель комплексных классических групп Ли. На самом деле, мы описываем явный вид уравнения Тоды (9), которое эквивалентно исходному уравнению

6) Заметим, что в этом случае $n-n_{1}$ с необходимостью является четным числом. 


$\left.\begin{array}{c|c|r|r|r}{[0]_{2 N}} & {\left[k_{1}\right]_{2 N}} & {\left[k_{1}+k_{2}\right]_{2 N}} & \ldots & {\left[\sum_{\alpha=1}^{p-1} k_{\alpha}\right]_{2 N}} \\ {[N]_{2 N}} & {\left[k_{1}+N\right]_{2 N}} & {\left[k_{1}+k_{2}+N\right]_{2 N}} & \ldots & {\left[\sum_{\alpha=1}^{p-1} k_{\alpha}+N\right]_{2 N}} \\ \hline-\left[k_{1}\right]_{2 N} & {[0]_{2 N}} & {\left[k_{2}\right]_{2 N}} & \ldots & {\left[\sum_{\alpha=2}^{p-1} k_{\alpha}\right]_{2 N}} \\ -\left[k_{1}+N\right]_{2 N} & {[N]_{2 N}} & {\left[k_{2}+N\right]_{2 N}} & \ldots & {\left[\sum_{\alpha=2}^{p-1} k_{\alpha}+N\right]_{2 N}} \\ \hline-\left[k_{1}+k_{2}\right]_{2 N} & -\left[k_{2}\right]_{2 N} & {[0]_{2 N}} & \ldots & {\left[\sum_{\alpha=3}^{p-1} k_{\alpha}\right]_{2 N}} \\ -\left[k_{1}+k_{2}+N\right]_{2 N} & -\left[k_{2}+N\right]_{2 N} & {[N]_{2 N}} & \ldots & {\left[\sum_{\alpha=3}^{p-1} k_{\alpha}+N\right]_{2 N}} \\ \hline \vdots & \vdots & \vdots & \ddots & \vdots \\ \hline-\left[\sum_{\alpha=1}^{p-1} k_{\alpha}\right]_{2 N} & -\left[\sum_{\alpha=2}^{p-1} k_{\alpha}\right]_{2 N} & -\left[\sum_{\alpha=3}^{p-1} k_{\alpha}\right]_{2 N} & \ldots & {[0]_{2 N}} \\ -\left[\sum_{\alpha=1}^{p-1} k_{\alpha}+N\right]_{2 N} & -\left[\sum_{\alpha=2}^{p-1} k_{\alpha}+N\right]_{2 N} & -\left[\sum_{\alpha=3}^{p-1} k_{\alpha}+N\right]_{2 N} & \ldots & {[N]_{2 N}}\end{array}\right)$

Рис. 2. Структура внешней $\mathbb{Z}_{2 N}$-градуировки $\mathfrak{g l}_{n}(\mathbb{C})$.

Тоды (1) для случая интегрируемых $\mathbb{Z}$-градуировок с конечномерными градуировочными подпространствами.

Пусть $G$ есть комплексная классическая группа Ли и $\mathfrak{g}$ - ее алгебра Ли. Выберем какую-нибудь $\mathbb{Z}_{M}$-градуировку $\mathfrak{g}$. Как было показано выше, с точностью до сопряжений любая $\mathbb{Z}_{M}$-градуировка комплексной классической алгебры Ли имеет структуру, изображенную либо на рис. 1, либо на рис. 2. Во всех случаях алгебра Ли $\mathfrak{g}_{[0]_{M}}$ и группа Ли $G_{0}$ образованы блок-диагональными матрицами, и можно параметризовать отображение $\gamma$ как

$$
\gamma=\left(\begin{array}{llll}
\Gamma_{1} & & & \\
& \Gamma_{2} & & \\
& & \ddots & \\
& & & \Gamma_{p}
\end{array}\right),
$$

где для каждого $\alpha=1, \ldots, p$ отображение $\Gamma_{\alpha}$ является отображением из $\mathcal{M}$ в группу Ли $G L_{n_{\alpha}}(\mathbb{C})$. Отображения $\Gamma_{\alpha}$, вообе говоря, не являются независимыми. Они удовлетворяют определенным ограничениям, налагаемым структурой рассматриваемой группы Ли.

Пусть $L$ есть положительное целое число такое, что градуировочные подпространства $\mathfrak{g}_{+[k]_{M}}$ и $\mathfrak{g}_{-[k]_{M}}$ тривиальны при $0<k<L$. Ясно, что если $x \in \mathfrak{g}_{+[L]_{M}}$, то только блоки $x_{\alpha, \alpha+1}, \alpha=1, \ldots, p-1$, и $x_{p 1}$ в блок-матричном представлении (15) могут быть отличны от нуля. Следовательно, отображение $c_{+}$имеет структуру, изображенную на рис. 3 , где для каждого $\alpha=1, \ldots, p-1$ отображение $C_{+\alpha}$ есть отображение из $\mathcal{M}$ в пространство комплексных $\left(n_{\alpha} \times n_{\alpha+1}\right)$-матриц, а $C_{+0}$ есть отображение из $\mathcal{M}$ в пространство комплексных $\left(n_{p} \times n_{1}\right)$-матриц. При этом считается, 


$$
\left(\begin{array}{ccccc}
0 & C_{+1} & & & \\
& 0 & \ddots & & \\
& & \ddots & \ddots & \\
& & & 0 & C_{+(p-1)} \\
C_{+0} & & & & 0
\end{array}\right)\left(\begin{array}{ccccc}
0 & & & & C_{-0} \\
C_{-1} & 0 & & & \\
& \ddots & \ddots & & \\
& & \ddots & 0 & \\
& & & C_{-(p-1)} & 0
\end{array}\right)
$$

Рис. 3. Каноническая структура отображения $c_{+}$.
Рис. 4. Каноническая структура отображения $c_{-}$.

что если какие-то блоки среди $x_{\alpha, \alpha+1}, \alpha=1, \ldots, p-1$, и $x_{p 1}$ в общем блок-матричном представлении (15) имеют градуировочный индекс, отличный от $+[L]_{M}$, то соответствующие блоки в блок-матричном представлении $c_{+}$есть нулевые матрицы.

На основании аналогичных рассуждений можно понять, что отображение $c_{-}$имеет структуру, изображенную на рис. 4 , где для каждого $\alpha=1, \ldots, p-1$ отображение $C_{-\alpha}$ есть отображение из $\mathcal{M}$ в пространство комплексных $\left(n_{\alpha+1} \times n_{\alpha}\right)$-матриц, а $C_{-0}$ - отображение из $\mathcal{M}$ в пространство комплексных $\left(n_{1} \times n_{p}\right)$-матриц. При этом предполагается, что если какие-нибудь из блоков $x_{\alpha+1, \alpha}, \alpha=1, \ldots, p-1$, и $x_{1 p}$ в общем блок-матричном представлении (15) имеют градуировочный индекс, отличный от $-[L]_{M}$, то соответствующие блоки в блок-матричном представлении $c_{-}$являются нулевыми матрицами.

Условия (10) означают, что

$$
\partial_{+} C_{-\alpha}=0, \quad \partial_{-} C_{+\alpha}=0, \quad \alpha=0, \ldots, p-1 .
$$

Кроме того, матрицы $C_{+\alpha}$ и $C_{-\alpha}$ должны удовлетворять некоторым ограничениям, следующим из структуры алгебры Ли g.

Предположим теперь, что $G$ есть группа Ли $G L_{n}(\mathbb{C})$, и мы используем $\mathbb{Z}_{M}$-градуировку $\mathfrak{g l}_{n}(\mathbb{C})$ типа I. Нетрудно показать, что тогда уравнение Тоды (9) для отображения $\gamma$ эквивалентно следующей системе уравнений для отображений $\Gamma_{\alpha}$ :

$$
\begin{aligned}
\partial_{+}\left(\Gamma_{1}^{-1} \partial_{-} \Gamma_{1}\right) & =-\Gamma_{1}^{-1} C_{+1} \Gamma_{2} C_{-1}+C_{-0} \Gamma_{p}^{-1} C_{+0} \Gamma_{1}, \\
\partial_{+}\left(\Gamma_{2}^{-1} \partial_{-} \Gamma_{2}\right) & =-\Gamma_{2}^{-1} C_{+2} \Gamma_{3} C_{-2}+C_{-1} \Gamma_{1}^{-1} C_{+1} \Gamma_{2}, \\
& \vdots \\
\partial_{+}\left(\Gamma_{p-1}^{-1} \partial_{-} \Gamma_{p-1}\right) & =-\Gamma_{p-1}^{-1} C_{+(p-1)} \Gamma_{p} C_{-(p-1)}+C_{-(p-2)} \Gamma_{p-2}^{-1} C_{+(p-2)} \Gamma_{p-1}, \\
\partial_{+}\left(\Gamma_{p}^{-1} \partial_{-} \Gamma_{p}\right) & =-\Gamma_{p}^{-1} C_{+0} \Gamma_{1} C_{-0}+C_{-(p-1)} \Gamma_{p-1}^{-1} C_{+(p-1)} \Gamma_{p} .
\end{aligned}
$$

Уравнения Тоды, ассоциированные с группой петель группы Ли $S L_{n}(\mathbb{C})$, в случае $\mathbb{Z}_{M}$-градуировок внутреннего типа имеют в действительности тот же вид (20), что и уравнения Тоды, ассоциированные с группой петель группы $G L_{n}(\mathbb{C})$. При этом отображения $\Gamma_{\alpha}$ должны удовлетворять условию $\prod_{\alpha=1}^{p} \operatorname{det} \Gamma_{\alpha}=1$. Если имеется решение уравнения Тоды, ассоциированного с группой петель группы $G L_{n}(\mathbb{C})$, то можно легко получить решение уравнения Тоды, ассоциированного с соответствующей 
группой петель группы $S L_{n}(\mathbb{C})$. Каждое решение уравнения Тоды, ассоциированного с группой петель группы $S L_{n}(\mathbb{C})$, может быть получено из решения уравнения Тоды, ассоциированного с соответствующей группой петель группы Ли $G L_{n}(\mathbb{C})$.

Пусть теперь $G$ есть либо группа Ли $S O_{n}(\mathbb{C})$, либо группа Ли $S p_{n}(\mathbb{C})$, и мы используем $\mathbb{Z}_{M}$-градуировку $\mathfrak{s o}_{n}(\mathbb{C})$ или $\mathfrak{s p}_{n}(\mathbb{C})$ типа I. Тогда мы получаем два различных случая. Для $p=2 s$ уравнение Тоды (9) эквивалентно системе

$$
\begin{aligned}
\partial_{+}\left(\Gamma_{1}^{-1} \partial_{-} \Gamma_{1}\right) & =-\Gamma_{1}^{-1} C_{+1} \Gamma_{2} C_{-1}+C_{-0}{ }^{J} \Gamma_{1} C_{+0} \Gamma_{1}, \\
\partial_{+}\left(\Gamma_{2}^{-1} \partial_{-} \Gamma_{2}\right) & =-\Gamma_{2}^{-1} C_{+2} \Gamma_{3} C_{-2}+C_{-1} \Gamma_{1}^{-1} C_{+1} \Gamma_{2}, \\
& \vdots \\
\partial_{+}\left(\Gamma_{s-1}^{-1} \partial_{-} \Gamma_{s-1}\right) & =-\Gamma_{s-1}^{-1} C_{+(s-1)} \Gamma_{s} C_{-(s-1)}+C_{-(s-2)} \Gamma_{s-2}^{-1} C_{+(s-2)} \Gamma_{s-1}, \\
\partial_{+}\left(\Gamma_{s}^{-1} \partial_{-} \Gamma_{s}\right) & =-\Gamma_{s}^{-1} C_{+s}{ }^{J}\left(\Gamma_{s}^{-1}\right) C_{-s}+C_{-(s-1)} \Gamma_{s-1}^{-1} C_{+(s-1)} \Gamma_{s},
\end{aligned}
$$

где $C_{+0}=-{ }^{J} C_{+0}, C_{-0}=-{ }^{J} C_{-0}$ и $C_{+s}=-{ }^{J} C_{+s}, C_{-s}=-{ }^{J} C_{-s}$ для ортогонального случая, тогда как $C_{+0}={ }^{J} C_{+0}, C_{-0}={ }^{J} C_{-0}$ и $C_{+s}={ }^{J} C_{+s}, C_{-s}={ }^{J} C_{-s}$ для симплек-

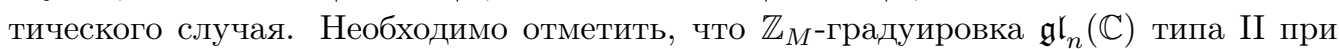
$p=2 s$ также дает уравнения (21). Однако в этом случае дополнительно к условиям ${ }^{J} C_{+0}=-C_{+0}$ и ${ }^{J} C_{-0}=-C_{-0}$ имеются условия ${ }^{J} C_{+s}=C_{+s}$ и ${ }^{J} C_{-s}=C_{-s}$.

Возвращаясь к $\mathbb{Z}_{M}$-градуировкам $\mathfrak{s o}_{n}(\mathbb{C})$ или $\mathfrak{s p}_{n}(\mathbb{C})$ типа I, можно видеть, что при $p=2 s-1$ уравнение Тоды (9) эквивалентно системе

$$
\begin{aligned}
\partial_{+}\left(\Gamma_{1}^{-1} \partial_{-} \Gamma_{1}\right) & =-\Gamma_{1}^{-1} C_{+1} \Gamma_{2} C_{-1}+C_{-0}{ }^{J} \Gamma_{1} C_{+0} \Gamma_{1}, \\
\partial_{+}\left(\Gamma_{2}^{-1} \partial_{-} \Gamma_{2}\right) & =-\Gamma_{2}^{-1} C_{+2} \Gamma_{3} C_{-2}+C_{-1} \Gamma_{1}^{-1} C_{+1} \Gamma_{2}, \\
& \vdots \\
\partial_{+}\left(\Gamma_{s-1}^{-1} \partial_{-} \Gamma_{s-1}\right) & =-\Gamma_{s-1}^{-1} C_{+(s-1)} \Gamma_{s} C_{-(s-1)}+C_{-(s-2)} \Gamma_{s-2}^{-1} C_{+(s-2)} \Gamma_{s-1}, \\
\partial_{+}\left(\Gamma_{s}^{-1} \partial_{-} \Gamma_{s}\right) & =-{ }^{B_{s}}\left(C_{-(s-1)} \Gamma_{s-1}^{-1} C_{+(s-1)} \Gamma_{s}\right)+C_{-(s-1)} \Gamma_{s-1}^{-1} C_{+(s-1)} \Gamma_{s} .
\end{aligned}
$$

Здесь ${ }^{B_{s}} \Gamma_{s}=\Gamma_{s}^{-1}$ с $B_{s}=J$ в ортогональном случае и $B_{s}=K$ в симплектическом случае. Находим также, что в ортогональном случае $C_{+0}=-{ }^{J} C_{+0}$ и $C_{-0}=-{ }^{J} C_{-0}$, тогда как в симплектическом случае получаем $C_{+0}={ }^{J} C_{+0}$ и $C_{-0}={ }^{J} C_{-0}$. Используя

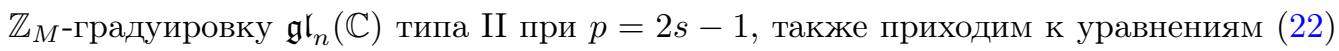
с $B_{s}=K$ и условиями $C_{+0}=-{ }^{J} C_{+0}, C_{-0}=-{ }^{J} C_{-0}$. При этом $\mathbb{Z}_{M}$-градуировка $\mathfrak{g l}_{n}(\mathbb{C})$ типа III для $p=2 s-1$ дает уравнения, эквивалентные уравнениям (22) с $B_{s}=J$ и $C_{+0}={ }^{J} C_{+0}, C_{-0}={ }^{J} C_{-0}$.

Наконец, если использовать $\mathbb{Z}_{M}$-градуировки $\mathfrak{s o}_{n}(\mathbb{C})$ или $\mathfrak{s p}_{n}(\mathbb{C})$ типа II, то видно, что уравнение Тоды (9) эквивалентно системе

$$
\begin{aligned}
\partial_{+}\left(\Gamma_{1}^{-1} \partial_{-} \Gamma_{1}\right) & =-\Gamma_{1}^{-1} C_{+1} \Gamma_{2} C_{-1}+{ }^{B_{1}}\left(\Gamma_{1}^{-1} C_{+1} \Gamma_{2} C_{-1}\right), \\
\partial_{+}\left(\Gamma_{2}^{-1} \partial_{-} \Gamma_{2}\right) & =-\Gamma_{2}^{-1} C_{+2} \Gamma_{3} C_{-2}+C_{-1} \Gamma_{1}^{-1} C_{+1} \Gamma_{2}, \\
& \vdots \\
\partial_{+}\left(\Gamma_{s-1}^{-1} \partial_{-} \Gamma_{s-1}\right) & =-\Gamma_{s-1}^{-1} C_{+(s-1)} \Gamma_{s} C_{-(s-1)}+C_{-(s-2)} \Gamma_{s-2}^{-1} C_{+(s-2)} \Gamma_{s-1}, \\
\partial_{+}\left(\Gamma_{s}^{-1} \partial_{-} \Gamma_{s}\right) & =-{ }^{B_{s}}\left(C_{-(s-1)} \Gamma_{s-1}^{-1} C_{+(s-1)} \Gamma_{s}\right)+C_{-(s-1)} \Gamma_{s-1}^{-1} C_{+(s-1)} \Gamma_{s} .
\end{aligned}
$$


Здесь ${ }^{B_{1}} \Gamma_{1}=\Gamma_{1}^{-1},{ }^{B} \Gamma_{s}=\Gamma_{s}^{-1}$ с $B_{1}=J, B_{s}=J$ в ортогональном случае и $B_{1}=K$, $B_{s}=K$ симплектическом случае. При этом $\mathbb{Z}_{M}$-градуировка $\mathfrak{g l}_{n}(\mathbb{C})$ типа III при $p=2 s-2$ дает уравнения (23) с $B_{1}=J, B_{s}=K$.

Следует отметить, что для каждого типа тодовских систем, рассмотренных выше, если для некоторого $\alpha$ оказывается, что $C_{+\alpha}=0$ или $C_{-\alpha}=0$, то рассматриваемая система уравнений становится тодовской системой, ассоциированной с соответствующей конечномерной группой Ли (см., например, работы [13], [14]). Следовательно, для того чтобы уравнения действительно были ассоциированы с некоторой группой петель, необходимо предположить, что все отображения $C_{+\alpha}$ и $C_{-\alpha}$ нетривиальны. Это возможно, только если $k_{\alpha}=L$ для каждого $\alpha=1, \ldots, p-1$ и $M=p L$. Без всякой потери общности можно считать, что $L=1$.

Уравнения (21)-(23) могут быть получены из уравнений (20) при помощи некоторой процедуры складывания, описанной в приложении. Эта процедура также помогает понять, что возможны только описанные здесь классы тодовских уравнений, ассоциированных с рассматриваемыми группами петель.

\section{5. ПРОСТЕЙШИЙ СЛУЧАЙ: НЕАБЕЛЕВЫ

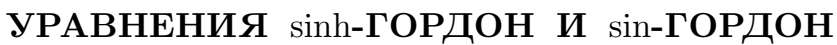

Определяя отображения $\Gamma_{\alpha}, C_{+\alpha}$ и $C_{-\alpha}$ для всех целочисленных значений индекса $\alpha$ с условиями периодичности $\Gamma_{\alpha+p}=\Gamma_{\alpha}, C_{-(\alpha+p)}=C_{-\alpha}$ и $C_{+(\alpha+p)}=C_{+\alpha}$, можно рассматривать систему (20) как бесконечную периодическую систему

$$
\partial_{+}\left(\Gamma_{\alpha}^{-1} \partial_{-} \Gamma_{\alpha}\right)=-\Gamma_{\alpha}^{-1} C_{+\alpha} \Gamma_{\alpha+1} C_{-\alpha}+C_{-(\alpha-1)} \Gamma_{\alpha-1}^{-1} C_{+(\alpha-1)} \Gamma_{\alpha}
$$

В частности, при $n=r p, n_{\alpha}=r, C_{-\alpha}=C_{+\alpha}=I_{r}$ приходим к уравнениям [23], [24]

$$
\partial_{+}\left(\Gamma_{\alpha}^{-1} \partial_{-} \Gamma_{\alpha}\right)=-\Gamma_{\alpha}^{-1} \Gamma_{\alpha+1}+\Gamma_{\alpha-1}^{-1} \Gamma_{\alpha}
$$

Полагая $p=2$, находим простейший набор уравнений Тоды:

$$
\partial_{+}\left(\Gamma_{1}^{-1} \partial_{-} \Gamma_{1}\right)=-\Gamma_{1}^{-1} \Gamma_{2}+\Gamma_{2}^{-1} \Gamma_{1}, \quad \partial_{+}\left(\Gamma_{2}^{-1} \partial_{-} \Gamma_{2}\right)=-\Gamma_{2}^{-1} \Gamma_{1}+\Gamma_{1}^{-1} \Gamma_{2},
$$

где $\Gamma_{1}$ и $\Gamma_{2}$ есть $(r \times r)$-матрицы. Эти уравнения инвариантны по отношению к преобразованиям $\Gamma_{1} \rightarrow{ }^{\mathrm{t}}\left(\Gamma_{2}^{-1}\right), \Gamma_{2} \rightarrow{ }^{\mathrm{t}}\left(\Gamma_{1}^{-1}\right)$, где верхний индекс t означает обычное транспонирование. Эта инвариантность говорит о возможности редукции к случаю $\Gamma_{1}={ }^{\mathrm{t}}\left(\Gamma_{2}^{-1}\right)=\Gamma$. При этом рассматриваемые уравнения сводятся к уравнению

$$
\partial_{+}\left(\Gamma^{-1} \partial_{-} \Gamma\right)=-\left({ }^{\mathrm{t}} \Gamma \Gamma\right)^{-1}+{ }^{\mathrm{t}} \Gamma \Gamma \text {. }
$$

С точки зрения возможных физических приложений интересно рассмотреть вещественные формы уравнений Тоды (9). Предположим, что заданы инволютивный антиголоморфный автоморфизм $\sigma$ группы $G$ и его компаньон для алгебры Ли, а также инволютивный антилинейный автоморфизм $\Sigma$ алгебры $\mathfrak{g}$, согласованный с $\mathbb{Z}$-градуировкой. Последнее означает, что если $x \in \mathfrak{g}_{[k]_{M}}$, то $\Sigma(x) \in \mathfrak{g}_{[k]_{M}}$. Предположим также, что $\Sigma\left(c_{+}\right)=c_{+}$и $\Sigma\left(c_{-}\right)=c_{-}$. В этом случае если $\gamma$ является 
решением уравнения Тоды (9), то $\sigma \circ \gamma$ тоже является решением этого уравнения ${ }^{7)}$, и непротиворечивая редукция к случаю $\sigma \circ \gamma=\gamma$ возможна.

Можно построить две неэквивалентные вещественные формы уравнения Тоды (24). Первая из них основана на некомпактной вещественной форме, задаваемой вещественными матрицами. Тогда $\sigma(\Gamma)=\Gamma^{*}=\Gamma$. Для $r=1$, полагая $\Gamma=e^{F}$, получаем уравнение sinh-Гордон

$$
\partial_{+} \partial_{-} F=2 \sinh F
$$

Другая форма уравнения Тоды основана на компактной вещественной форме, задаваемой унитарными матрицами. Тогда $\sigma(\Gamma)=\left(\Gamma^{\dagger}\right)^{-1}=\Gamma$, и уравнение Тоды $(24)$ удобно записать в виде

$$
\partial_{+}\left(\Gamma^{-1} \partial_{-} \Gamma\right)=-\left({ }^{\mathrm{t}} \Gamma \Gamma\right)^{\dagger}+{ }^{\mathrm{t}} \Gamma \Gamma \text {. }
$$

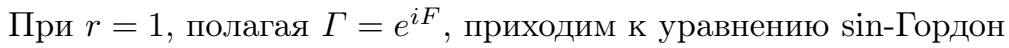

$$
\partial_{+} \partial_{-} F=2 \sin F
$$

\section{6. ЗАКЛЮЧЕНИЕ}

Итак, существуют четыре неэквивалентных класса уравнений Тоды, ассоциированных с группами петель комплексных классических групп Ли, если их алгебры Ли наделены интегрируемыми $\mathbb{Z}$-градуировками с конечномерными градуировочными подпространствами. Первый класс представлен уравнениями (20). Это основной класс петлевых уравнений Тоды, в котором нет никаких ограничений на отображения $\Gamma_{\alpha}$ и $C_{ \pm \alpha}$.

Напротив, три других класса петлевых уравнений Тоды, представленных здесь уравнениями (21)-(23), получаются из уравнений (20) при наложении некоторых ограничений на отображения $\Gamma_{\alpha}$ и $C_{ \pm \alpha}$. Эти ограничения следуют из соотношений, определяющих рассматриваемую группу или алгебру, а также из типа градуировки. Интересно, например, что все эти три класса петлевых уравнений Тоды возникают также в общем линейном случае для градуировок внешнего типа, хотя при этом изначально нет никаких групповых и алгебраических условий.

Результаты классификации можно пояснить некоторой графической процедурой складывания (см. приложение).

\section{ПРИЛОЖЕНИЕ}

\section{Графическое представление петлевых уравнений Тоды}

Здесь мы описываем графическое представление уравнений Тоды, ассоциированных с группами петель. Это представление может оказаться полезным для простого объяснения того факта, что на самом деле возникают только четыре неэквивалентных класса петлевых уравнений Тоды.

\footnotetext{
7) При этом мы полагаем, что $\mathcal{M}=\mathbb{R}^{2}$.
} 
Мы используем следующие правила отождествления, проиллюстрированные рис. 5. Каждое отображение $\Gamma_{\alpha}$, входящее в соотношение (18), изображается маленьким кружком на окружности, а отображение $C_{ \pm \alpha}-$ дугой между двумя такими кружками, уже отождествленными с $\Gamma_{\alpha}$ и $\Gamma_{\alpha+1}$. Нумерация по индексу $\alpha$ идет вдоль окружности в направлении против часовой стрелки. Вся тодовская система соответствует окружности с $p$ прикрепленными маленькими кружками.
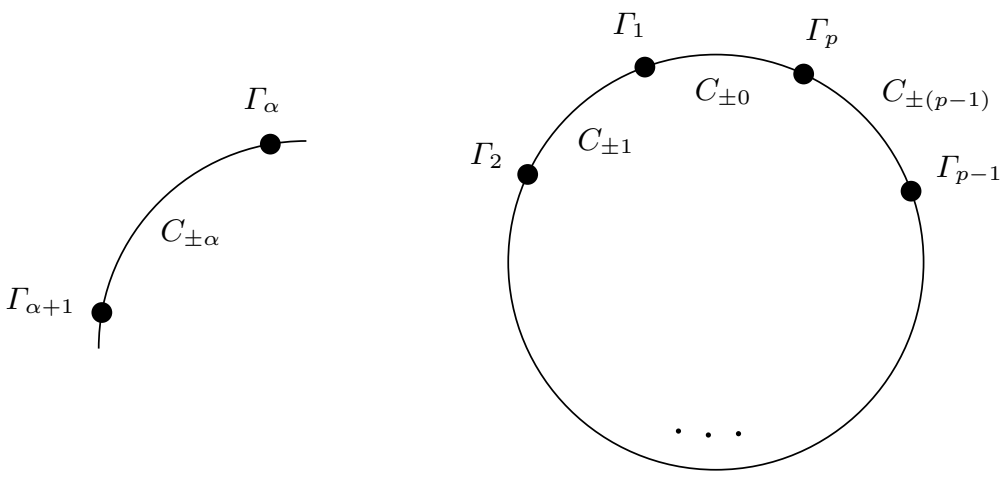

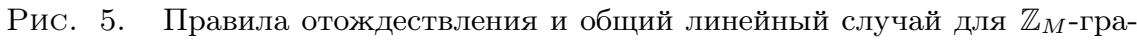
дуировок внутреннего типа: уравнения Тоды (20).

Для групп петель общих линейных групп Ли, когда используются $\mathbb{Z}_{M}$-градуировки внутреннего типа, нет никаких дополнительных ограничений на отображения $\gamma, c_{+}$и $c_{-}$, кроме их канонических форм, заданных соотношением (18) и рис. $3,4$. В этом случае мы останавливаемся на рис. 5, изображающем уравнения Тоды (20).

Существенно другая ситуация возникает для других классических групп Ли при использовании внутренних и внешних $\mathbb{Z}_{M}$-градуировок и для общей линейной группы при использовании $\mathbb{Z}_{M}$-градуировок внешнего типа.

Учет влияния условий, определяющих группу и алгебру Ли, на отображения $\gamma$ и $c_{+}, c_{-}$, соответственно, приводит к необходимости сложить размеченную окружность вдоль ее диаметра, помеченного пунктирной линией на рис. 6-8. Тодовская система согласована со своим графическим представлением, если каждый объект на окружности, отождествленный с $\Gamma_{\alpha}$ и $C_{ \pm \alpha}, \alpha=1, \ldots, p$, находит своего компаньона на другой стороне окружности при таком складывании.

Ясно, что возможны только три принципиально различных ситуации: две для четного $p$ (рис. 6,7$)$, и одна для нечетного $p$, (рис. 8а). Дополнительно обведенными кружками изображаются те, которые складываются сами с собой. Рис. $8 б$ описывает другой вариант складывания. Он соответствует уравнениям Тоды, которые могут быть получены из уравнений (22) подстановками $\Gamma_{\alpha} \rightarrow{ }^{B_{s}}\left(\Gamma_{s-\alpha+1}^{-1}\right), C_{ \pm \alpha} \rightarrow{ }^{J} C_{ \pm(s-\alpha)}$, дополненными заменой $B_{s} \rightarrow B_{1}$.

Наконец, интересно провести сравнение со случаем тодовских систем, ассоциированных с конечномерными группами Ли [13], [14]. Рассматривая аналогичное графическое представление на прямой и учитывая, при этом нет никакой периодич- 


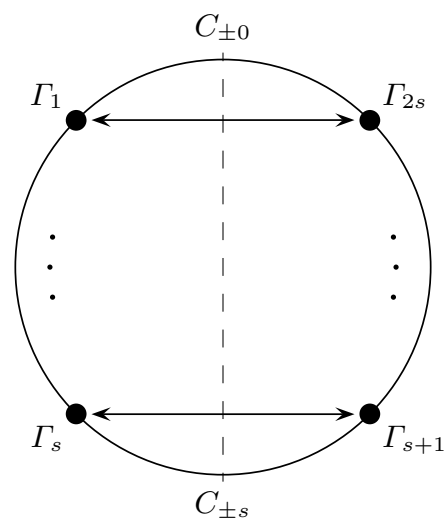

Рис. 6. Число $p=2 s$. Уравнения Тоды (21), где ${ }^{J} C_{ \pm 0}=\varepsilon C_{ \pm 0}$ и ${ }^{J} C_{ \pm s}=\varepsilon C_{ \pm s}$.

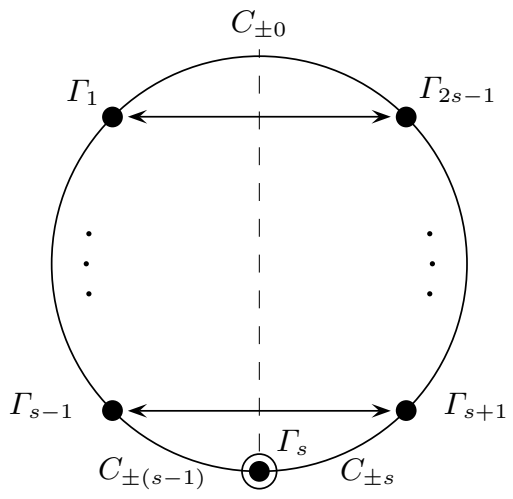

$a$

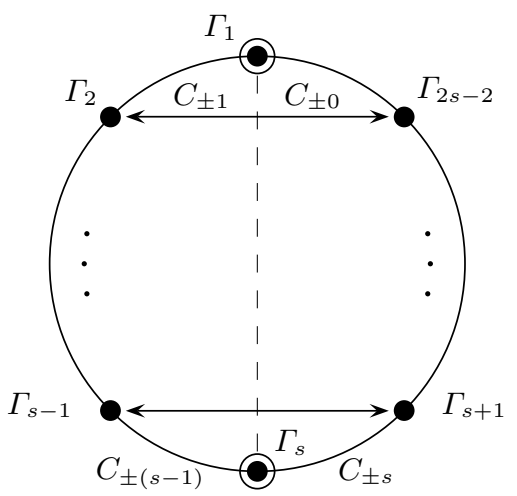

Рис. 7. Число $p=2 s-2$. Уравнения Тоды (23), где ${ }^{B_{1}} \Gamma_{1}=\Gamma_{1}^{-1}$ и ${ }^{B_{s}} \Gamma_{s}=\Gamma_{s}^{-1}$.

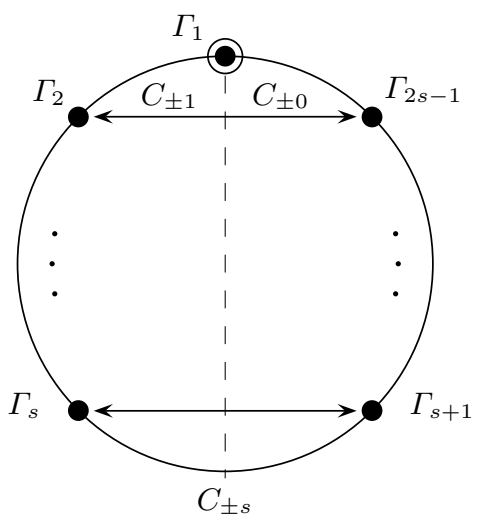

б

Рис. 8. Число $p=2 s-1$. Уравнения Тоды $(22)$, где ${ }^{B_{s}} \Gamma_{s}=\Gamma_{s}^{-1}$ и ${ }^{J} C_{ \pm 0}=\varepsilon C_{ \pm 0}$, и эквивалентные уравнения Тоды с ${ }^{B_{1}} \Gamma_{1}=\Gamma_{1}^{-1}$ и ${ }^{J} C_{ \pm s}=\varepsilon C_{ \pm s}$. Здесь $\varepsilon$ есть либо + , либо -.

ности по индексам, можно видеть, что в дополнение к общему линейному случаю возникают только два различных класса тодовских систем (один для четного $p$ и один для нечетного $p$ ), ассоциированных с ортогональной и симплектической группами Ли.

Благодарности. Настоящая работа была частично поддержана РФФИ (грант № 07-01-00234). 
Х. С. НИРОВ, А. В. РАЗУМОВ

\section{Список литературы}

[1] А.Н. Лезнов, М. В. Савельев, Групповые методы интегрирования нелинейных динамических систем, Наука, М., 1985.

[2] A.V. Razumov, M.V. Saveliev, Comm. Anal. Geom., 2 (1994), 461-511; arXiv: hep-th/9311167.

[3] A. V. Razumov, M. V. Saveliev, Lie Algebras, Geometry, and Toda-type Systems, Cambridge Lecture Notes in Phys., 8, Cambridge Univ. Press, Cambridge, 1997.

[4] A. N. Leznov, "The internal symmetry group and methods of field theory for integrating exactly soluble dynamic systems", Group Theoretical Methods in Physics, Proc. Zvenigorod seminar (Zvenigorod, 1982), Harwood, New York, 1985, 443-457.

[5] J.-L. Gervais, M. V. Saveliev, Nucl. Phys. B, 453:1-2 (1995), 449-476; arXiv: hep-th/9505047.

[6] L. A. Ferreira, J.-L. Gervais, J. Sánchez Guillén, M. V. Saveliev, Nucl. Phys. B, 470:1-2 (1996), 236-288; arXiv: hep-th/9512105.

[7] A. G. Bueno, L. A. Ferreira, A. V. Razumov, Nucl. Phys. B, 626:3 (2002), 463-499; arXiv: hep-th/0105078.

[8] A. V. Razumov, M. V. Saveliev, "On some class of multidimensional nonlinear integrable systems", Proc. II Int. A. D. Sakharov Conf. on Physics (Moscow, 1996), eds. I. M. Dremin, A. M. Semikhatov, World Scientific, Singapore, 1997, 547-551; arXiv: hep-th/9607017.

[9] А. В. Разумов, М. В. Савельев, ТМФ, 112:2 (1997), 254-282; arXiv: hep-th/9609031.

[10] L. A. Ferreira, J. L. Miramontes, J. Sánchez Guillén, Nucl. Phys. B, 449:3 (1995), 631-679; arXiv: hep-th/9412127.

[11] C. R. Fernández-Pousa, M. V. Gallas, T. J. Hollowood, J. L. Miramontes, Nucl. Phys. B, 484:3 (1997), 609-630; arXiv: hep-th/9606032.

[12] A. V. Razumov, M. V. Saveliev, Nucl. Phys. B, 494:3 (1997), 657-686; arXiv: hep-th/9612081.

[13] A. V. Razumov, M.V. Saveliev, A. B. Zuevsky, "Non-abelian Toda equations associated with classical Lie groups", Symmetries and Integrable Systems, ed. A. N. Sissakian, JINR, Dubna, 1999, 190-203; arXiv: math-ph/9909008.

[14] Kh. S. Nirov, A. V. Razumov, "On classification of non-abelian Toda systems", Geometrical an Topological Ideas in Modern Physics, ed. V. A. Petrov, IHEP, Protvino, 2002, 213-221; arXiv: nlin.SI/0305023.

[15] Э. Пресли, Г. Сигал, Группы петель, Мир, М., 1990.

[16] Kh.S. Nirov, A.V. Razumov, Comm. Math. Phys., 267:3 (2006), 587-610; arXiv: math-ph/0504038.

[17] Kh.S. Nirov, A. V. Razumov, Nucl. Phys. B, 782:3 (2007), 241-275; arXiv: math-ph/0612054.

[18] R. Hamilton, Bull. Amer. Math. Soc., 7:1 (1982), 65-222.

[19] J. Milnor, "Remarks on infinite-dimensional Lie groups", Relativity, Groups and Topology II, eds. B. S. DeWitt, R. Stora, North-Holland, Amsterdam, 1984, 1007-1057.

[20] А.Л. Онищик, Е.Б. Винберг, Семинар по группам Ли и алгебраическим группам, Наука, М., 1988.

[21] В. Г. Кац, Бесконечномерные алгебры Ли, Мир, М., 1993.

[22] Э.Б. Винберг, В.В. Горбацевич, А.Л. Онищик, Группы Ли и алгебры Ли 3, Итоги науки и техн., Соврем. пробл. мат. Фундам. направления, 41, ВИНИТИ, М., 1990.

[23] И. М. Кричевер, Приложение к статье: Б. А. Дубровин, "Тэта-функции и нелинейные уравнения", УМН, 36:2(218) (1981), 72-77.

[24] A. V. Mikhailov, Physica D, 3:1-2 (1981), 73-117. 